Hydrologic and Water-Quality Data at Selected Sites in the Upper Animas River Watershed, Southwestern Colorado, 1997-99

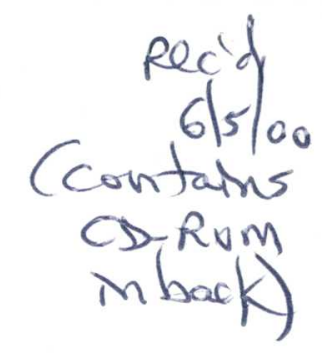

Open-File Report 00-53

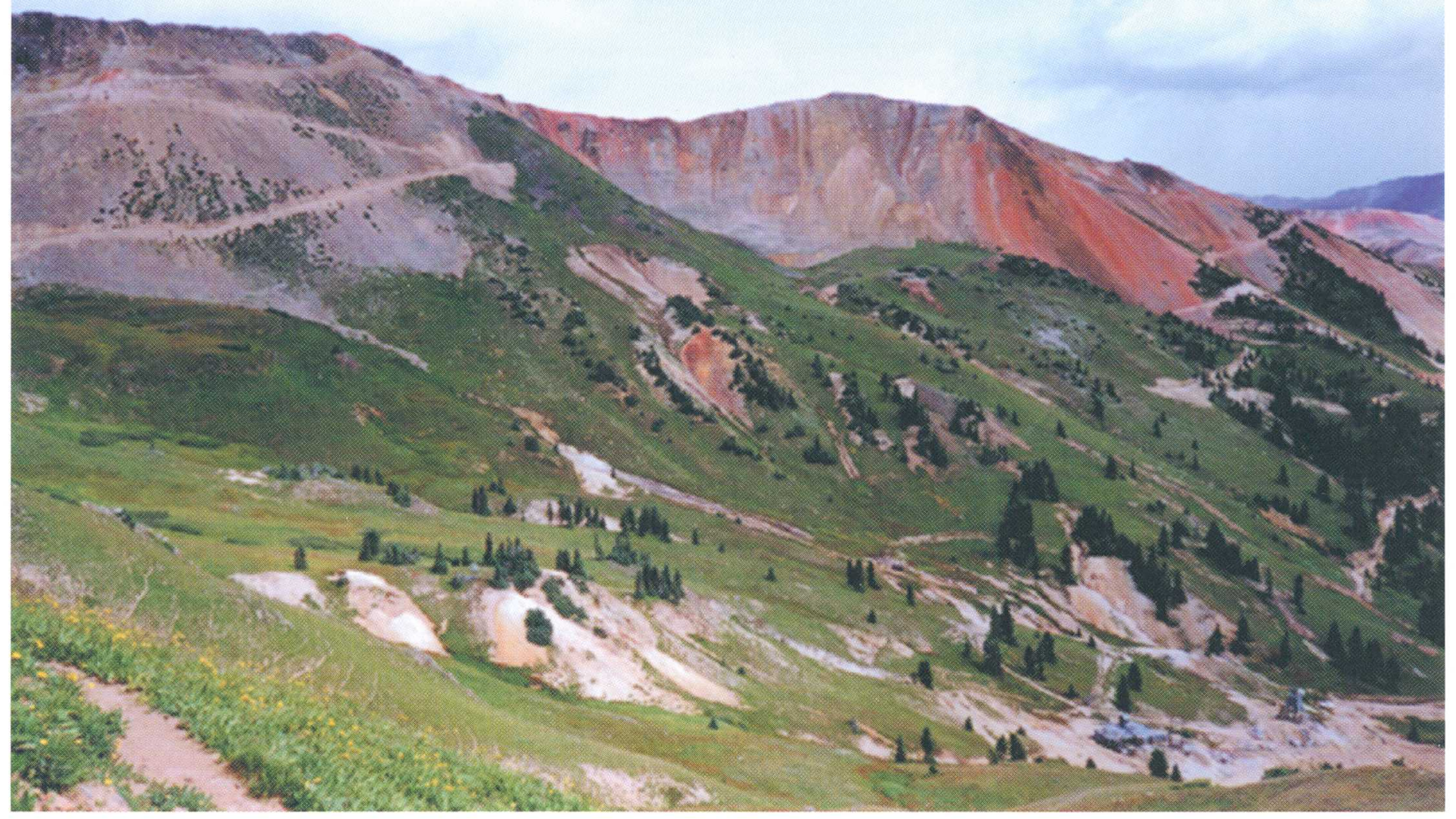

U.S. Department of the Interior

U.S. Geolgical Survey 


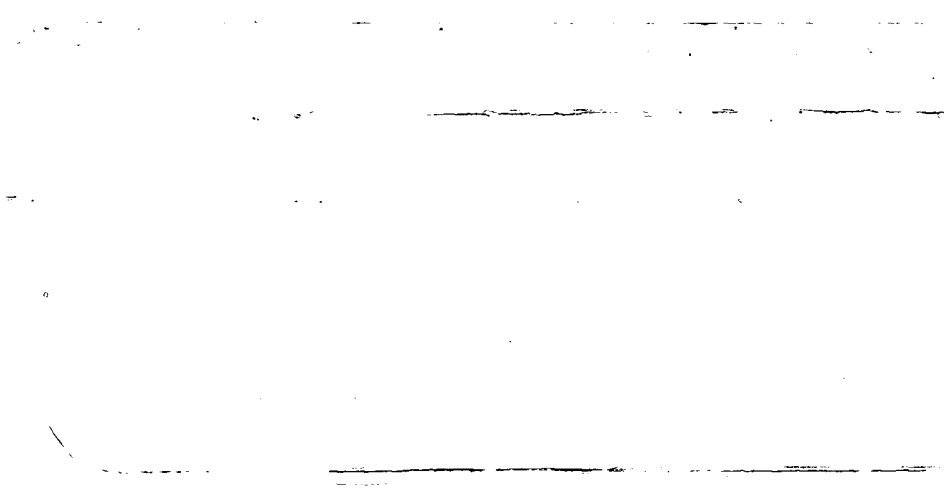

L............... 


\section{Hydrologic and Water-Quality Data at Selected Sites in the Upper Animas River Watershed, Southwestern Colorado, 1997-99}

By M. Alisa Mast, Jonathan B. Evans, Kenneth J. Leib, and Winfield G. Wright

U.S. GEOLOGICAL SURVEY

Open-File Report 00-53 


\section{U.S. DEPARTMENT OF THE INTERIOR \\ BRUCE BABBITT, Secretary}

U.S. GEOLOGICAL SURVEY

Charles G. Groat, Director

The use of firm, trade, and brand names in this report is for identification purposes only and does not constitute endorsement by the U.S. Geological Survey.

For additional information write to:

District Chief

U.S. Geological Survey

Box 25046, Mail Stop 415

Denver Federal Center

Denver, CO 80225-0046
Copies of this report can be purchased from:

U.S. Geological Survey

Information Services

Box 25286

Federal Center

Denver, CO 80225 


\section{CONTENTS}

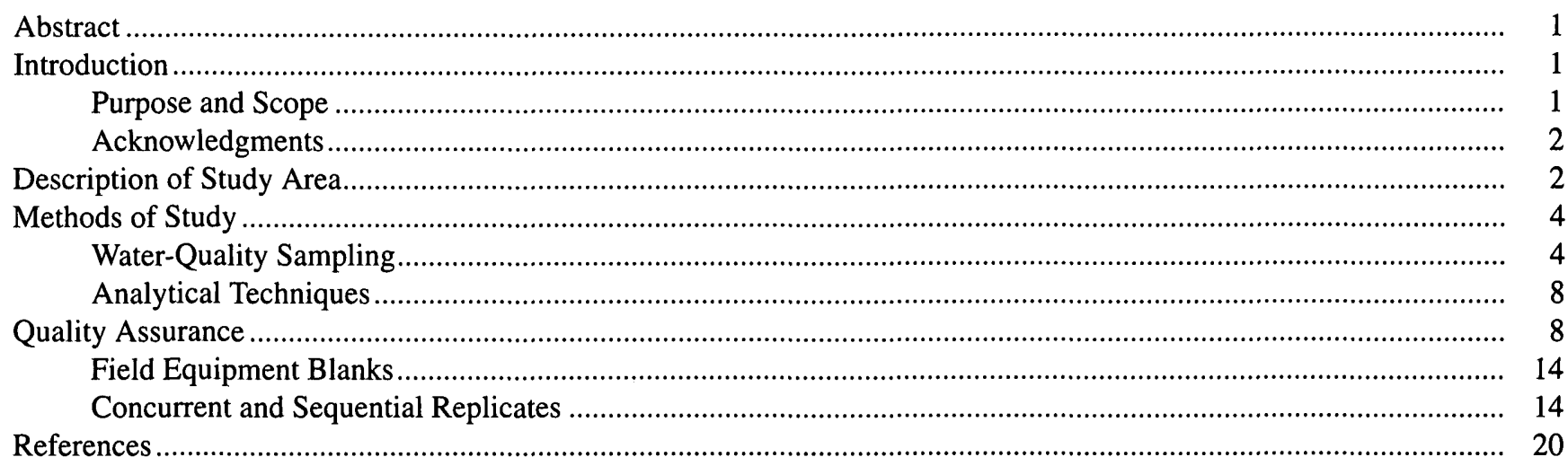

\section{PLATE}

[Plate is in pocket attached to inside back cover]

1. Map of upper Animas River watershed and locations of water-quality sampling sites at selected springs and streams in southwestern Colorado

\section{FIGURES}

1. Map of upper Animas River watershed and locations of four USGS streamflow-gaging stations and nine water-quality sampling sites along the main stem and major tributaries

2. Map of upper Animas River watershed and locations of water-quality sampling sites at selected mines

\section{TABLES}

1. Streamflow-gaging stations and water-quality sampling sites on the main stem and major tributaries in the upper Animas River watershed

2. Water-quality sampling sites at selected mines in the upper Animas River watershed

3. Water-quality sampling sites at selected springs and streams in the upper Animas River watershed.........

4. Analytical techniques and corresponding detection limits used for water-quality analyses

5. Chemical analyses of field equipment blanks collected during the study period....

6. Comparison of chemical analyses of environmental samples and sequential replicates collected during the study period.

\section{1} 1

7. Comparison of chemical analyses of environmental samples and concurrent replicates collected at the Animas River below Silverton during the study period .

[CD-ROM containing tables 8-28 and abbreviations is in pocket attached to inside front cover]

Hydrologic and water-quality data tables and abbreviations

8-19. Daily mean streamflow for:

8. Animas River below Silverton, Colorado, in water year 1997 .................................................................... 22

9. Animas River below Silverton, Colorado, in water year 1998 ....................................................................... 23

10. Animas River below Silverton, Colorado, in water year 1999 ................................................................... 24

11. Animas River at Silverton, Colorado, in water year 1997 ........................................................................ 25

12. Animas River at Silverton, Colorado, in water year 1998 .......................................................................... 26

13. Animas River at Silverton, Colorado, in water year 1999...................................................................... 27

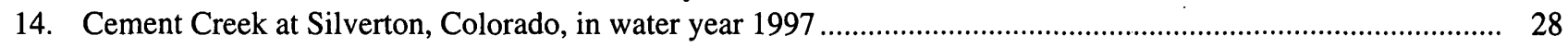

15. Cement Creek at Silverton, Colorado, in water year 1998 ..................................................................... 29

16. Cement Creek at Silverton, Colorado, in water year 1999 ............................................................................ 30 


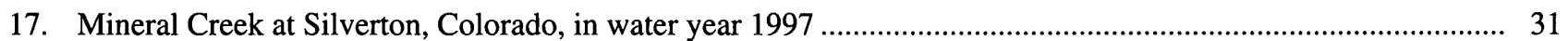

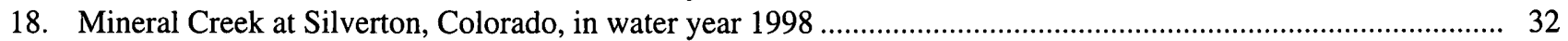

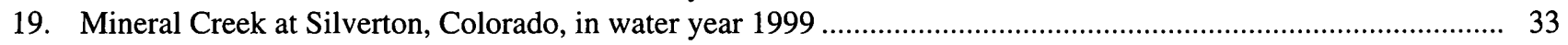

20-28. Water-quality data for:

20. Animas River below Silverton, Colorado, 1997-99 .................................................................................. 34

21. Animas River at Silverton, Colorado, 1997-99 ........................................................................................... 37

22. Cement Creek at Silverton, Colorado, 1997-99 ........................................................................................ 40

23. Mineral Creek at Silverton, Colorado, 1997-99 ...................................................................................... 43

24. Nine sites along the main-stem upper Animas River and two major tributaries, 1998-99 ................................ 46

25. Selected mine sites in the upper Animas River watershed, 1997-99 ............................................................ 50

26. Selected streams and springs in the subbasin above upper Animas River at Silverton, Colorado ........................ 59

27. Selected streams and springs in the subbasin above Cement Creek at Silverton, Colorado................................ 61

28. Selected streams and springs in the subbasin above Mineral Creek at Silverton, Colorado ............................... 65

\section{CONVERSION FACTORS AND ABBREVIATIONS}

\begin{tabular}{rll}
\hline Multiply & By & To obtain \\
\hline inch & 2.54 & centimeter \\
cubic foot per second $\left(\mathrm{ft}^{3} / \mathrm{s}\right)$ & 0.02832 & cubic meter per second \\
mile $(\mathrm{mi})$ & 1.609 & kilometer \\
liter $(\mathrm{L})$ & 0.2641 & gallons $(\mathrm{US})$ \\
foot $(\mathrm{ft})$ & 0.3048 & meter \\
foot per mile $\left(\mathrm{ft} / \mathrm{mi}^{2}\right)$ & 0.189 & meter per kilometer \\
square mile $\left(\mathrm{mi}^{2}\right)$ & 2.59 & square kilometer \\
\hline
\end{tabular}

Temperature in degrees Fahrenheit $\left({ }^{\circ} \mathrm{F}\right)$ may be converted to degrees Celsius $\left({ }^{\circ} \mathrm{C}\right)$ using the following equation:

$$
{ }^{\circ} \mathrm{C}=\left({ }^{\circ} \mathrm{F}-32\right) / 1.8
$$

Additional abbreviations used in this report:

micrometer $(\mu \mathrm{m})$

microsiemens per centimeter at 25 degrees Celsius $(\mu \mathrm{S} / \mathrm{cm})$

milligram per liter $(\mathrm{mg} / \mathrm{L})$

microgram per liter $(\mu \mathrm{g} / \mathrm{L})$

milliliter $(\mathrm{mL})$

millimeter (mm) 


\title{
Hydrologic and Water-Quality Data at Selected Sites in the Upper Animas River Watershed, Southwestern Colorado, 1997-99
}

\author{
By M. Alisa Mast, Jonathan B. Evans, Kenneth J. Leib, and Winfield G. Wright
}

\begin{abstract}
The water quality and aquatic resources of the upper Animas River watershed are affected by more than 1,500 abandoned mines from historical metal-mining activities in the late 1800's and early 1900's. In 1997, the U.S. Geological Survey implemented the Abandoned Mine Lands Initiative to provide scientific information to Federal land-management agencies responsible for remediation of abandoned mine sites on publicly owned land. This report presents hydrologic and water-quality data collected as part of the Abandoned Mine Lands Initiative during water years 1997-99 in the upper Animas River watershed, Colorado. Water-quality samples were collected from four streamflow-gaging stations, nine sites along the main stem and two major tributaries, 55 abandoned mine sites, and 194 selected stream and spring sites. Data include field measurements of streamflow, specific conductance, $\mathrm{pH}$, water temperature, and dissolved oxygen and laboratory analyses of major inorganic and selected traceelement constituents. Daily mean discharge values are tabulated for the four streamflowgaging stations. A CD-ROM containing hydrologic and water-quality data is in the pocket of this report.
\end{abstract}

\section{INTRODUCTION}

The upper Animas River watershed (UARW) lies in the southwestern part of the Colorado mineral belt and was heavily mined in the late 1800's and early 1900 's for gold, silver, zinc, and lead. Numerous prospects, draining mine adits, waste-rock piles, and mill tailings left behind by historical mining activities have severely affected the water quality and aquatic resources of the Animas River and its tributaries. Recently, there has been much interest by local stakeholders, and by State and Federal agencies, in remediating abandoned mine sites in the watershed with the goal of restoring aquatic ecosystems along some reaches of the Animas River. Effective and costefficient remediation of abandoned mine lands requires knowledge of the degree of contamination of the environment as well as an understanding of the processes by which abandoned mine lands disturb the natural ecosystem. In 1997, the U.S. Geological Survey (USGS) implemented the Abandoned Mine Lands Initiative (AMLI) to provide this type of scientific information to the Federal land-management agencies responsible for remediation of abandoned mine sites in the UARW (Nimick and von Guerard, 1998). This is part of a larger strategy by the U.S. Department of the Interior and the U.S. Department of Agriculture to coordinate activities between Federal and State agencies and local stakeholders for the cleanup of Federal lands affected by abandoned mine lands. One of the main components of the AMLI is to describe the geological, hydrological, and biological characteristics of the watershed. This information will be used to improve understanding of the processes that control the occurrence and transport of contaminants and to identify mining sites that have the most adverse effect on the water quality and aquatic resources of the Animas River.

\section{Purpose and Scope}

The purpose of this report is to present hydrologic and water-quality data and methods of data 
collection at selected sampling sites in the UARW in southwestern Colorado (fig. 1). Data were collected during water years 1997-99 as part of the AMLI. Bimonthly to weekly water-quality samples were collected at four streamflow-gaging stations and nine sites along the main-stem upper Animas River and two major tributaries to monitor seasonal variations in stream chemistry and solute fluxes in the watershed. The four streamflow-gaging stations are the Animas River below Silverton (station 09359020; A72), Animas River at Silverton (station 09358000; A68), Cement Creek at Silverton (station 09358550; C48), and Mineral Creek at Silverton (station 09359010; M34). One hundred and thirty-eight water-quality samples were collected at 55 selected mine sites during the study period to characterize spatial and seasonal variability in mine-water chemistry and discharge. Water-quality samples at 194 selected streams and springs were sampled throughout the watershed during summer base-flow conditions to help distinguish natural from mining-related sources of metals to surface water. Data presented in this report include field measurements of streamflow, specific conductance, $\mathrm{pH}$, water temperature, and dissolved oxygen and laboratory analyses of concentrations of major inorganic and selected trace-element constituents. Daily mean discharge values for streamflow also are presented for the four streamflow-gaging stations. Supplemental data are on the CD-ROM in the pocket of the report cover.

\section{Acknowledgments}

Many aspects of this study would not have been possible without the support of the Upper Animas River Stakeholders Group; the U.S. Department of the Interior, Bureau of Land Management and Bureau of Reclamation; and the U.S. Department of Agriculture Forest Service. The authors are grateful for assistance and guidance from USGS employees Greg Alexander, James Bennett, Mark Gress, Dave Grey, Paul von Guerard, David Roth, Jenny A. Taylor, and Philip Verplanck.

\section{DESCRIPTION OF STUDY AREA}

The upper Animas River watershed is in the San Juan Mountains in southwestern Colorado. The river drains $146 \mathrm{mi}^{2}$ of rugged mountainous terrain ranging in elevation from 9,200 $\mathrm{ft}$ near the town of Silverton, Colorado, to $13,894 \mathrm{ft}$ at the summit of Vermilion Peak (fig. 1). The upper Animas River is a southwardflowing tributary of the San Juan River and has a channel length of about $15.5 \mathrm{mi}$ above the streamflowgaging station at Animas River below Silverton (A72) and an average stream gradient of about $184 \mathrm{ft} / \mathrm{mi}$. The main channel of the river is perennial, and mean monthly discharge ranges from about $63 \mathrm{ft}^{3} / \mathrm{s}$ in February to $1,196 \mathrm{ft}^{3} / \mathrm{s}$ in June. Snowmelt is the major hydrologic event of the year, and more than 60 percent of the annual streamflow occurs in May, June, and July. Average annual runoff for the watershed was 28 inches from 1992 to 1998 (Crowfoot and others, 1999). The Animas River has two major tributaries, Cement Creek and Mineral Creek, which together account for about 50 percent of the annual discharge from the watershed. Climate of the area is characterized by long, cold winters and short (3-4 months), cool summers. Average monthly air temperature at Silverton, Colorado, ranges from $16.0^{\circ} \mathrm{F}$ in January to $55.3^{\circ} \mathrm{F}$ in July (URL http://www.wrcc.sage.dri.edu, accessed March 2000). Precipitation averages 45 inches annually, of which about 70 percent accumulates in a seasonal snowpack between November and April (Wirt and others, 1999). Most of the remaining precipitation falls during monsoonal thundershowers in late summer and early fall. The watershed lies in the Southern Rocky Mountain Forest ecoregion, and much of the area is above treeline, which is at an average elevation of $11,600 \mathrm{ft}$. The lower slopes are covered by a dense forest of Engelmann spruce and subalpine fir (Bailey and others, 1994).

The UARW lies within the San Juan volcanic field of Tertiary age, which includes the San Juan, Uncompahgre, and Silverton calderas that formed about 28 million years ago (Lipman and others, 1976; Bove and others, 1999). Most of the bedrock in the study area consists of thick sequences of volcanic rocks that are composed of lava flows, ash-flow tuffs, tuff breccias, conglomerates, and mudflow breccias (Lipman and others, 1973; Ringrose, 1982). The lavas and tuffs generally are of intermediate composition and are composed of plagioclase, biotite, augite, quartz, and hornblende. Nearly all the volcanic rocks in the study area were subject to some degree of hydrothermal alteration after deposition (Burbank and Luedke, 1969). Hydrothermal activity related to the Silverton caldera caused regional propylitization of the volcanic rocks, which altered the rocks to chlorite and 


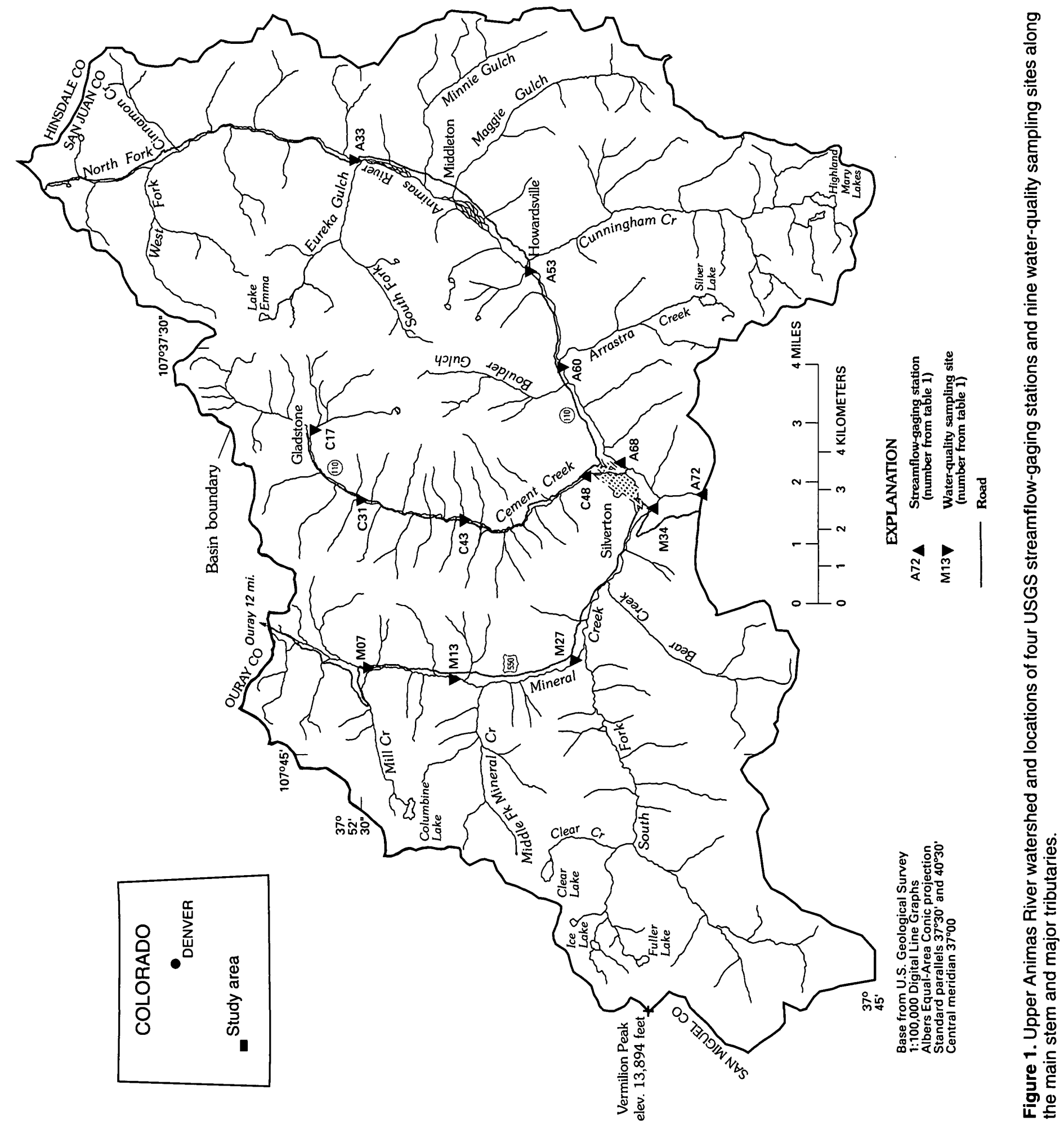


epidote with calcite and pyrite often disseminated throughout the groundmass. The propylitized rocks were subsequently mineralized and hydrothermally altered during three later episodes of magmatism (11-23 million years ago), which were the source of most ore deposition in the area (Casadevall and Ohmoto, 1977). Most economic ore deposits occur in polymetallic vein systems that follow structures formed during subsidence and resurgence of the San Juan, Uncompahgre, and Silverton calderas (Burbank and Luedke, 1969; Ringrose, 1982). The area was first prospected for precious metals in 1860, and most mining activities occurred in the late 1800's and early 1900 's. Production was primarily from vein type deposits that yielded gold, silver, zinc, and lead. Over 1,500 prospect pits, abandoned mines, and dismantled mill sites are now scattered throughout the watershed as a result of past mining activities.

\section{METHODS OF STUDY}

This section includes a description of the methods used to collect and analyze water-quality samples collected in the UARW as part of the AMLI. Methods of operation for streamflow-gaging stations are standardized within the USGS (Rantz and others, 1982) and will not be included in this report. Daily mean streamflow values at the four streamflow-gaging stations for 1997-99 are presented in the "Hydrologic and Water-Quality Data" section of this report and also are in Crowfoot and others (1998), Crowfoot and others (1999), and Crowfoot and others (U.S. Geological Survey, written commun., 2000).

\section{Water-Quality Sampling}

Water-quality samples were collected seasonally (weekly to bimonthly) at the four streamflow-gaging stations and at nine upstream sites along the main stem Animas River and major tributaries. The sites are listed in table 1 and locations shown in figure 1. Seasonal (monthly to bimonthly) samples also were collected at seven mine sites: the Avalanche Mine, Bandora Mine, Bonner Mine, Elk Tunnel, Evelyn Mine, Forest Queen Mine, and Mighty Monarch Mine. The mine sites are listed in table 2 and locations shown in figure 2. Field measurements at the sampling sites included stream discharge, specific conductance, $\mathrm{pH}$, water temperature, and dissolved oxygen. Waterquality measurements were made using methods

Table 1. Streamflow-gaging stations and water-quality sampling sites on the main stem and major tributaries in the upper Animas River watershed

[Site locations are shown in figure 1; identification number is USGS downstream order number; elevation in feet; watershed area in square miles; ,-- not reported]

\begin{tabular}{|c|c|c|c|c|c|c|}
\hline $\begin{array}{c}\text { Site } \\
\text { number }\end{array}$ & Site name & $\begin{array}{c}\text { Identification } \\
\text { number }\end{array}$ & Latitude & Longitude & Elevation & $\begin{array}{c}\text { Watershed } \\
\text { area }\end{array}$ \\
\hline A72 & Animas River below Silverton, Colorado & 09359020 & $37^{\circ} 47^{\prime} 25^{\prime \prime}$ & $107^{\circ} 40^{\prime} 01^{\prime \prime}$ & 9,200 & 146 \\
\hline A68 & Animas River at Silverton, Colorado & 09358000 & $37^{\circ} 48^{\prime} 40^{\prime \prime}$ & $107^{\circ} 39^{\prime} 31^{\prime \prime}$ & 9,290 & 70.6 \\
\hline $\mathrm{C} 48$ & Cement Creek at Silverton, Colorado & 09358550 & $37^{\circ} 49^{\prime} 11^{\prime \prime}$ & $107^{\circ} 39^{\prime} 47^{\prime \prime}$ & 9,380 & 20.1 \\
\hline M34 & Mineral Creek at Silverton, Colorado & 09359010 & $37^{\circ} 48^{\prime} 10^{\prime \prime}$ & $107^{\circ} 40^{\prime} 20^{\prime \prime}$ & 9,245 & 52.5 \\
\hline A33 & Animas River at Eureka & -- & $37^{\circ} 52^{\prime} 45^{\prime \prime}$ & $107^{\circ} 33^{\prime} 55^{\prime \prime}$ & 9,852 & 18.2 \\
\hline A53 & Animas River at Howardsville & -- & $37^{\circ} 50^{\prime} 07^{\prime \prime}$ & $107^{\circ} 35^{\prime} 52^{\prime \prime}$ & 9,642 & 57.7 \\
\hline A60 & Animas River below Arrastra Gulch & -- & $37^{\circ} 49^{\prime} 38^{\prime \prime}$ & $107^{\circ} 37^{\prime} 34^{\prime \prime}$ & 9,475 & 60.0 \\
\hline $\mathrm{C} 17$ & South Fork Cement Creek at Gladstone & -- & $37^{\circ} 53^{\prime} 21^{\prime \prime}$ & $107^{\circ} 39^{\prime} 10^{\prime \prime}$ & 10,469 & 3.1 \\
\hline $\mathrm{C} 31$ & Cement Creek at Fairview Gulch Bridge & -- & $37^{\circ} 52^{\prime} 30^{\prime \prime}$ & $107^{\circ} 40^{\prime} 16^{\prime \prime}$ & 10,206 & 9.4 \\
\hline $\mathrm{C} 43$ & Cement Creek near Yukon Mine & -- & $37^{\circ} 50^{\prime} 56^{\prime \prime}$ & $107^{\circ} 40^{\prime} 35^{\prime \prime}$ & 9,894 & 14.8 \\
\hline M07 & Mineral Creek at Chattanooga & -- & $37^{\circ} 52^{\prime} 27^{\prime \prime}$ & $107^{\circ} 43^{\prime} 26^{\prime \prime}$ & 10,240 & 4.4 \\
\hline M13 & Mineral Creek at Burro Bridge & -- & $37^{\circ} 51^{\prime} 02^{\prime \prime}$ & $107^{\circ} 43^{\prime} 31^{\prime \prime}$ & 10,006 & 10.4 \\
\hline M27 & Mineral Creek above South Fork & -- & $37^{\circ} 49^{\prime} 16^{\prime \prime}$ & $107^{\circ} 13^{\prime} 08^{\prime \prime}$ & 9,534 & 20.0 \\
\hline
\end{tabular}


Table 2. Water-quality sampling sites at selected mines in the upper Animas River watershed

[Site locations are shown in figure 2; elevation in feet; site MS24 eliminated from this report]

\begin{tabular}{|c|c|c|c|c|}
\hline $\begin{array}{c}\text { Site } \\
\text { number }\end{array}$ & Site name & Latitude & Longitude & Elevation \\
\hline MS1 & Anglo Saxon Mine & $37^{\circ} 51^{\prime} 32^{\prime \prime}$ & $107^{\circ} 40^{\prime} 36^{\prime \prime}$ & 10,080 \\
\hline MS2 & Avalanche Mine & $37^{\circ} 52^{\prime} 24^{\prime \prime}$ & $107^{\circ} 40^{\prime} 07^{\prime \prime}$ & 10,540 \\
\hline MS3 & Bagley Tunnel & $37^{\circ} 55^{\prime} 59^{\prime \prime}$ & $107^{\circ} 34^{\prime} 50^{\prime \prime}$ & 11,440 \\
\hline MS4 & Bandora Mine & $37^{\circ} 47^{\prime} 12^{\prime \prime}$ & $107^{\circ} 48^{\prime} 04^{\prime \prime}$ & 10,784 \\
\hline MS5 & Big Colorado Mine & $37^{\circ} 52^{\prime} 37^{\prime \prime}$ & $107^{\circ} 38^{\prime} 46^{\prime \prime}$ & 11,060 \\
\hline MS6 & Black Hawk Mine & $37^{\circ} 52^{\prime} 56^{\prime \prime}$ & $107^{\circ} 38^{\prime} 05^{\prime \prime}$ & 11,600 \\
\hline MS7 & Bonner Mine & $37^{\circ} 50^{\prime} 41^{\prime \prime}$ & $107^{\circ} 44^{\prime} 09^{\prime \prime}$ & 10,040 \\
\hline MS8 & Brooklyn Mine & $37^{\circ} 51^{\prime} 37^{\prime \prime}$ & $107^{\circ} 42^{\prime} 54^{\prime \prime}$ & 11,348 \\
\hline MS9 & Bullion King Mine & $37^{\circ} 53^{\prime} 19^{\prime \prime}$ & $107^{\circ} 44^{\prime} 32^{\prime \prime}$ & 12,400 \\
\hline MS10 & Burbank Mine & $37^{\circ} 48^{\prime} 43^{\prime \prime}$ & $107^{\circ} 46^{\prime} 37^{\prime \prime}$ & 10,480 \\
\hline MS11 & Elk Tunnel & $37^{\circ} 52^{\prime} 13^{\prime \prime}$ & $107^{\circ} 40^{\prime} 29^{\prime \prime}$ & 10,200 \\
\hline MS12 & Esmeralda Mine & $37^{\circ} 50^{\prime} 54^{\prime \prime}$ & $107^{\circ} 31^{\prime} 57^{\prime \prime}$ & 11,490 \\
\hline MS13 & Evelyn Mine & $37^{\circ} 53^{\prime} 17^{\prime \prime}$ & $107^{\circ} 39^{\prime} 53^{\prime \prime}$ & 10,580 \\
\hline MS14 & Ferricrete Mine & $37^{\circ} 52^{\prime} 05^{\prime \prime}$ & $107^{\circ} 43^{\prime} 35^{\prime \prime}$ & 10,300 \\
\hline MS15 & Forest Queen Mine & $37^{\circ} 51^{\prime} 58^{\prime \prime}$ & $107^{\circ} 33 ' 54^{\prime \prime}$ & 9,860 \\
\hline MS16 & Grand Mogul Mine & $37^{\circ} 54^{\prime} 36^{\prime \prime}$ & $107^{\circ} 37^{\prime} 49^{\prime \prime}$ & 11,760 \\
\hline MS17 & Imogene Mine & $37^{\circ} 51^{\prime} 45^{\prime \prime}$ & $107^{\circ} 43^{\prime} 41^{\prime \prime}$ & 10,360 \\
\hline MS18 & Independence Mine & $37^{\circ} 50^{\prime} 41^{\prime \prime}$ & $107^{\circ} 44^{\prime 2} 3^{\prime \prime}$ & 10,180 \\
\hline MS19 & Joe and Johns Mine & $37^{\circ} 53^{\prime} 31^{\prime \prime}$ & $107^{\circ} 40^{\prime} 42^{\prime \prime}$ & 11,200 \\
\hline MS20 & Kittimac Mine & $37^{\circ} 51^{\prime} 54^{\prime \prime}$ & $107^{\circ} 32^{\prime} 12^{\prime \prime}$ & 11,600 \\
\hline MS21 & Lark Mine & $37^{\circ} 53^{\prime} 34^{\prime \prime}$ & $107^{\circ} 40^{\prime} 50^{\prime \prime}$ & 11,320 \\
\hline MS22 & London Mine & $37^{\circ} 56^{\prime} 57^{\prime \prime}$ & $107^{\circ} 35^{\prime} 01^{\prime \prime}$ & 11,920 \\
\hline MS23 & Lucky Jack Mine & $37^{\circ} 57^{\prime} 14^{\prime \prime}$ & $107^{\circ} 34^{\prime} 21^{\prime \prime}$ & 12,000 \\
\hline MS25 & Mighty Monarch Mine & $37^{\circ} 48^{\prime} 29^{\prime \prime}$ & $107^{\circ} 39^{\prime} 24^{\prime \prime}$ & 9,477 \\
\hline MS26 & Mine in upper Cement Creek & $37^{\circ} 54^{\prime} 39^{\prime \prime}$ & $107^{\circ} 38^{\prime} 00^{\prime \prime}$ & 11,680 \\
\hline MS27 & Mine in Bighorn Gulch & $37^{\circ} 52^{\prime} 28^{\prime \prime}$ & $107^{\circ} 41^{\prime} 43^{\prime \prime}$ & 12,400 \\
\hline MS28 & Mine east of Chattanooga beaver ponds & $37^{\circ} 52^{\prime} 15^{\prime \prime}$ & $107^{\circ} 43^{\prime} 07^{\prime \prime}$ & 10,600 \\
\hline MS29 & Mine in South Fork Eureka Gulch & $37^{\circ} 52^{\prime} 35^{\prime \prime}$ & $107^{\circ} 36^{\prime} 06^{\prime \prime}$ & 11,015 \\
\hline MS30 & Mine in Georgia Gulch & $37^{\circ} 53^{\prime} 03^{\prime \prime}$ & $107^{\circ} 41^{\prime} 13^{\prime \prime}$ & 11,900 \\
\hline MS31 & Mine in gulch south of Hancock Gulch & $37^{\circ} 50^{\prime} 12^{\prime \prime}$ & $107^{\circ} 39^{\prime} 46^{\prime \prime}$ & 10,440 \\
\hline MS32 & Mine in Middle Fork Cement Creek & $37^{\circ} 53^{\prime} 04^{\prime \prime}$ & $107^{\circ} 38^{\prime} 28^{\prime \prime}$ & 11,060 \\
\hline MS33 & Mine in Minnie Gulch & $37^{\circ} 51^{\prime} 47^{\prime \prime}$ & $107^{\circ} 33^{\prime} 09^{\prime \prime}$ & 10,480 \\
\hline MS34 & Mine near Paradise Portal & $37^{\circ} 50^{\prime} 32^{\prime \prime}$ & $107^{\circ} 45^{\prime} 52^{\prime \prime}$ & 10,600 \\
\hline MS35 & Mine in Prospect Gulch & $37^{\circ} 53^{\prime} 27^{\prime \prime}$ & $107^{\circ} 41^{\prime} 17^{\prime \prime}$ & 11,640 \\
\hline MS36 & Mine in upper Animas River & $37^{\circ} 53^{\prime} 56^{\prime \prime}$ & $107^{\circ} 33^{\prime 2} 7^{\prime \prime}$ & 10,400 \\
\hline MS37 & Mine in upper Browns Gulch & $37^{\circ} 51^{\prime} 46^{\prime \prime}$ & $107^{\circ} 42^{\prime} 22^{\prime \prime}$ & 11,840 \\
\hline MS38 & Mine in upper Cement Creek & $37^{\circ} 54^{\prime} 27^{\prime \prime}$ & $107^{\circ} 38^{\prime} 22^{\prime \prime}$ & 11,280 \\
\hline
\end{tabular}


Table 2. Water-quality sampling sites at selected mines in the upper Animas River watershed-Continued [Site locations are shown in figure 2; elevation in feet; site MS24 eliminated from this report]

\begin{tabular}{llllc}
\hline $\begin{array}{c}\text { Site } \\
\text { number }\end{array}$ & \multicolumn{1}{c}{ Site name } & Latitude & Longitude & Elevation \\
\hline MS39 & Mine in upper Minnie Gulch & $37^{\circ} 50^{\prime} 17^{\prime \prime}$ & $107^{\circ} 31^{\prime} 33^{\prime \prime}$ & 12,000 \\
MS40 & Mine in upper North Fork Cement Creek & $37^{\circ} 53^{\prime} 50^{\prime \prime}$ & $107^{\circ} 37^{\prime} 37^{\prime \prime}$ & 12,650 \\
MS41 & Mine above Brooklyn \#6 & $37^{\circ} 51^{\prime} 53^{\prime \prime}$ & $107^{\circ} 42^{\prime} 35^{\prime \prime}$ & 11,890 \\
MS42 & Mine near Horseshoe Bend & $37^{\circ} 52^{\prime} 26^{\prime \prime}$ & $107^{\circ} 43^{\prime} 58^{\prime \prime}$ & 10,420 \\
MS43 & Mine near Hwy 550 south of Middle Fork & $37^{\circ} 50^{\prime} 25^{\prime \prime}$ & $107^{\circ} 43^{\prime} 31^{\prime \prime}$ & 10,080 \\
& Mineral Creek & & & \\
MS44 & Mine northwest of Burro Bridge & $37^{\circ} 51^{\prime} 20^{\prime \prime}$ & $107^{\circ} 43^{\prime} 27^{\prime \prime}$ & 10,440 \\
MS45 & Mine near McMillan Peak & $37^{\circ} 52^{\prime} 46^{\prime \prime}$ & $107^{\circ} 41^{\prime} 43^{\prime \prime}$ & 12,450 \\
MS46 & Mine west of Burro Bridge & $37^{\circ} 51^{\prime} 02^{\prime \prime}$ & $107^{\circ} 43^{\prime} 45^{\prime \prime}$ & 10,200 \\
MS47 & Mine near Eureka Gulch & $37^{\circ} 53^{\prime} 07^{\prime \prime}$ & $107^{\circ} 35^{\prime} 28^{\prime \prime}$ & 10,680 \\
MS48 & Mine near Burro Bridge & $37^{\circ} 51^{\prime} 01^{\prime \prime}$ & $107^{\circ} 43^{\prime} 30^{\prime \prime}$ & 10,040 \\
MS49 & Old Hundred Mine & $37^{\circ} 49^{\prime} 24^{\prime \prime}$ & $107^{\circ} 35^{\prime} 04^{\prime \prime}$ & 9,980 \\
MS50 & Queen Anne Mine & $37^{\circ} 54^{\prime} 52^{\prime \prime}$ & $107^{\circ} 37^{\prime} 47^{\prime \prime}$ & 12,200 \\
MS51 & Silver Crown Mine & $37^{\circ} 52^{\prime} 18^{\prime \prime}$ & $107^{\circ} 44^{\prime} 35^{\prime \prime}$ & 11,800 \\
MS52 & Silver Ledge Mine & $37^{\circ} 52^{\prime} 36^{\prime \prime}$ & $107^{\circ} 38^{\prime} 38^{\prime \prime}$ & 10,970 \\
MS53 & Silver Wing Mine & $37^{\circ} 54^{\prime} 12^{\prime \prime}$ & $107^{\circ} 33^{\prime} 19^{\prime \prime}$ & 10,440 \\
MS54 & Treasure Mountain Mine & $37^{\circ} 54^{\prime} 49^{\prime \prime}$ & $107^{\circ} 34^{\prime} 11^{\prime \prime}$ & 11,640 \\
MS55 & Vermilion Mine & $37^{\circ} 56^{\prime} 10^{\prime \prime}$ & $107^{\circ} 36^{\prime} 01^{\prime \prime}$ & 12,400 \\
MS56 & Yukon Mine & $37^{\circ} 50^{\prime} 58^{\prime \prime}$ & $107^{\circ} 40^{\prime} 32^{\prime \prime}$ & 9,985 \\
\hline & & & \\
\hline
\end{tabular}




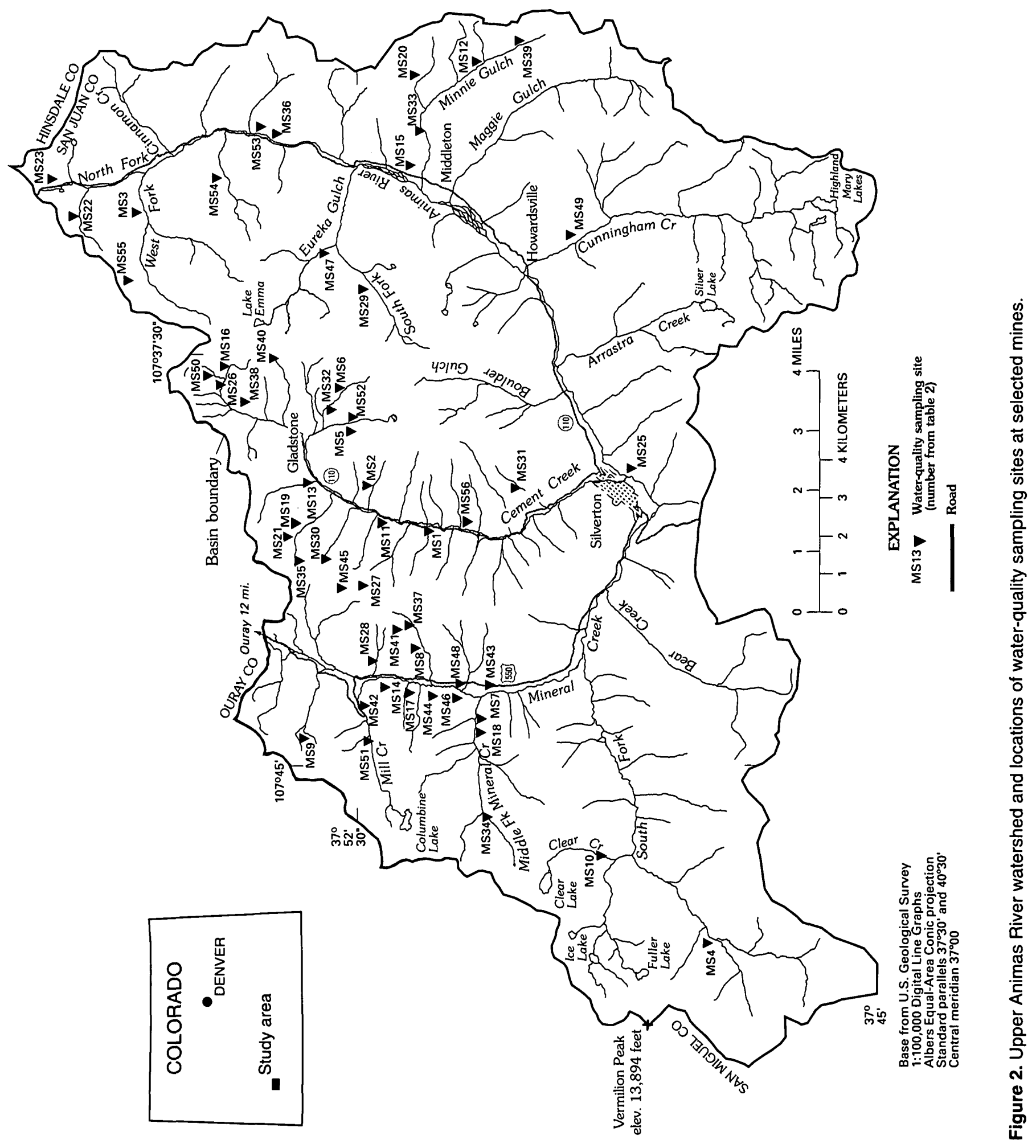


similar to those described by Wilde and Radtke (1998). Field meters were calibrated in the morning on the day of the sample collection. The $\mathrm{pH}$ meters were calibrated with $\mathrm{pH}-2$ and $\mathrm{pH}-4$ buffers or $\mathrm{pH}-4$ and $\mathrm{pH}-7$ buffers to bracket the range of $\mathrm{pH}$ values measured in the field. Specific-conductance (SC) meters were calibrated with two standards that bracketed the range of values expected at the sampling sites. Dissolved oxygen (DO) meters were calibrated to the saturated DO of water according to the water temperature and barometric pressure at each sampling site. The DO meters also were checked against a solution with a DO concentration of $0 \mathrm{mg} / \mathrm{L}$. Instantaneous discharge at the time of sampling was determined from the stage reading and stage-discharge-rating table at the four streamflow-gaging stations. At the nine upstream sites and seven mine sites, instantaneous discharge was measured using a current meter according to methods described by Rantz and others (1982). Standard USGS sampling techniques as described in Ward and Hair (1990) and Shelton (1994) were used to collect water-quality samples. Waterquality samples at the stream sites were collected using depth-integrating samplers utilizing the equalwidth-increments (EWI) method (Edwards and Glysson, 1988) then transferred into precleaned 2-L or 3-L polyethylene bottles. Mine-site samples were collected in precleaned polyethylene bottles by using the dip method.

Filtering and processing of water samples was done in a field laboratory in Silverton, Colorado, within 5 hours of sample collection. Standard USGS equipment (Horowitz and others, 1994) was used. Samples requiring filtered water were passed through a $0.45-\mu \mathrm{m}$ cellulose filter housed in a $47-\mathrm{mm}$ polycarbonate filtering unit using a peristaltic pump. The filter housing and pump tubing were soaked in dilute nitric acid and rinsed six times with deionized water between samples. Occasionally samples containing substantial amounts of sediment or colloids, were filtered using a $0.45-\mu \mathrm{m}$ capsule filter instead of the plate filter. Filtered and unfiltered aliquots for dissolved and total major inorganic and trace-element constituents were preserved with concentrated ultrapure nitric acid ( $1 \mathrm{~mL}$ per $250 \mathrm{~mL}$ of sample). A filtered unpreserved aliquot was collected for sulfate analysis, and an unfiltered unpreserved aliquot was collected for the alkalinity determination. For selected samples, a filtered aliquot was preserved with 6 molar hydrochloric acid for ferrous iron analysis. Collection and processing procedures similar to those described above were used for the remaining mine sites listed in table 2 and the selected spring and stream sites listed in table 3 and shown in plate 1, except that samples were filtered and processed at the sampling site rather than in the laboratory.

\section{Analytical Techniques}

Analytical techniques used for water-quality analyses and the corresponding analytical detection limits are listed in table 4 . Alkalinity was determined by incremental titration on unfiltered samples with $\mathrm{pH}$ greater than 4.5. The analysis was conducted at the sampling site or within 5 hours of sample collection at a USGS field laboratory in Silverton, Colorado. Major inorganic and selected trace-element constituents were analyzed on filtered and unfiltered (raw) samples by inductively coupled plasma-atomic emission spectroscopy (ICP-AES) and potassium was analyzed by atomic absorption spectroscopy (AA) at a USGS research laboratory in Boulder, Colorado. The unfiltered samples were digested in the laboratory with concentrated hydrochloride acid at $60 \mathrm{C}$ for 8 hours according to the method of Hoffman and others (1996). The digested samples were filtered through $0.45-\mu \mathrm{m}$ polycarbonate filters into acid-washed polyethylene bottles prior to ICP-AES and AA analysis. Sulfate was determined by a turbidimetric method using a Hach SulfoVer reagent and ferrous iron $\left(\mathrm{Fe}^{+2}\right)$ was determined by a colorimetric method using a Hach 1, 10-phenanthroline reagent. The sulfate and $\mathrm{Fe}^{+2}$ determinations were measured on filtered samples using a Hach DR2000 spectrophotometer with a $25-\mathrm{mL}$ sample cell. The quality of all laboratory analyses was assessed through the analysis of laboratory blanks, sample duplicates, USGS standard reference water samples (Long and Farrar, 1995), and calculated ion balances.

\section{QUALITY ASSURANCE}

Quality-assurance procedures used during the study period included daily calibration of waterquality meters, cleaning of sampling equipment and rinsing of sample bottles, and collection of qualitycontrol samples (field equipment blanks and replicate samples). Results of 15 field equipment blanks and 
Table 3. Water-quality sampling sites at selected springs and streams in the upper Animas River watershed (grouped according to three major subbasins of the watershed)

[UA, upper Animas River above Silverton; CC, Cement Creek; MC, Mineral Creek; site locations are shown on plate 1]

\begin{tabular}{|c|c|c|c|c|}
\hline $\begin{array}{c}\text { Site } \\
\text { number }\end{array}$ & Site name & Latitude & Longltude & $\begin{array}{c}\begin{array}{c}\text { Elevation } \\
\text { (feet) }\end{array}\end{array}$ \\
\hline UA1 & Boulder Gulch near mouth & $37^{\circ} 49^{\prime} 44^{\prime \prime}$ & $107^{\circ} 38^{\prime} 09^{\prime \prime}$ & 9,680 \\
\hline UA2 & Burrows Gulch above North Fork Animas River & $37^{\circ} 56^{\prime} 44^{\prime \prime}$ & $107^{\circ} 34^{\prime} 29^{\prime \prime}$ & 11,640 \\
\hline UA3 & Burrows Gulch below London Mine & $37^{\circ} 56^{\prime} 51^{\prime \prime}$ & $107^{\circ} 35^{\prime} 02^{\prime \prime}$ & 11,850 \\
\hline UA4 & Burrows Gulch below wetland & $37^{\circ} 56^{\prime} 49^{\prime \prime}$ & $107^{\circ} 34^{\prime} 39^{\prime \prime}$ & 11,800 \\
\hline UA5 & California Gulch above Vermillion Mine & $37^{\circ} 55^{\prime} 52^{\prime \prime}$ & $107^{\circ} 35^{\prime} 58^{\prime \prime}$ & 11,760 \\
\hline UA6 & California Gulch above Mountain Queen Mine & $37^{\circ} 54^{\prime} 55^{\prime \prime}$ & $107^{\circ} 36^{\prime} 48^{\prime \prime}$ & 12,400 \\
\hline UA7 & California Gulch below Mountain Queen Mine & $37^{\circ} 54^{\prime} 59^{\prime \prime}$ & $107^{\circ} 36^{\prime} 46^{\prime \prime}$ & 12,300 \\
\hline UA8 & Cinnamon Creek near mouth & $37^{\circ} 55^{\prime} 29^{\prime \prime}$ & $107^{\circ} 33^{\prime} 49^{\prime \prime}$ & 10,850 \\
\hline UA9 & Cunningham Gulch near mouth & $37^{\circ} 50^{\prime} 10^{\prime \prime}$ & $107^{\circ} 35^{\prime} 48^{\prime \prime}$ & 9,642 \\
\hline UA10 & Eureka Gulch above mines & $37^{\circ} 54^{\prime} 19^{\prime \prime}$ & $107^{\circ} 36^{\prime} 51^{\prime \prime}$ & 12,480 \\
\hline UA11 & Eureka Gulch above sink holes & $37^{\circ} 53^{\prime} 42^{\prime \prime}$ & $107^{\circ} 36^{\prime} 30^{\prime \prime}$ & 11,900 \\
\hline UA12 & Eureka Gulch below cut dike & $37^{\circ} 53^{\prime} 59^{\prime \prime}$ & $107^{\circ} 36^{\prime} 56^{\prime \prime}$ & 12,210 \\
\hline UA13 & Eureka Gulch below Terry Tunnel & $37^{\circ} 53^{\prime} 28^{\prime \prime}$ & $107^{\circ} 35^{\prime} 52^{\prime \prime}$ & 11,190 \\
\hline UA14 & Eureka Gulch near mouth & $37^{\circ} 52^{\prime} 48^{\prime \prime}$ & $107^{\circ} 34^{\prime} 10^{\prime \prime}$ & 9,890 \\
\hline UA15 & Grouse Gulch near mouth & $37^{\circ} 55^{\prime} 02^{\prime \prime}$ & $107^{\circ} 33^{\prime} 15^{\prime \prime}$ & 11,000 \\
\hline UA16 & Hematite Gulch near mouth & $37^{\circ} 50^{\prime} 09^{\prime \prime}$ & $107^{\circ} 35^{\prime} 52^{\prime \prime}$ & 9,648 \\
\hline UA17 & Horseshoe Creek near mouth & $37^{\circ} 57^{\prime} 00^{\prime \prime}$ & $107^{\circ} 34^{\prime} 18^{\prime \prime}$ & 11,750 \\
\hline UA18 & California Gulch below mines & $37^{\circ} 55^{\prime} 54^{\prime \prime}$ & $107^{\circ} 35^{\prime} 37^{\prime \prime}$ & 11,640 \\
\hline UA19 & Maggie Gulch near mouth & $37^{\circ} 51^{\prime} 18^{\prime \prime}$ & $107^{\circ} 34^{\prime} 31^{\prime \prime}$ & 9,711 \\
\hline UA20 & Maggie Gulch above mines & $37^{\circ} 48^{\prime} 47^{\prime \prime}$ & $107^{\circ} 32^{\prime} 10^{\prime \prime}$ & 11,840 \\
\hline UA21 & McCarty Basin & $37^{\circ} 53^{\prime} 32^{\prime \prime}$ & $107^{\circ} 36^{\prime} 14^{\prime \prime}$ & 11,560 \\
\hline UA22 & Minnie Gulch above Esmeralda Mine & $37^{\circ} 50^{\prime} 52^{\prime \prime}$ & $107^{\circ} 31^{\prime} 56^{\prime \prime}$ & 11,550 \\
\hline UA23 & Minnie Gulch near mouth & $37^{\circ} 51^{\prime} 44^{\prime \prime}$ & $107^{\circ} 34^{\prime} 19^{\prime \prime}$ & 9,760 \\
\hline UA24 & Niagara Gulch near mouth & $37^{\circ} 52^{\prime} 57^{\prime \prime}$ & $107^{\circ} 33^{\prime} 41^{\prime \prime}$ & 10,000 \\
\hline UA25 & North Fork Animas River above Burrows Gulch & $37^{\circ} 56^{\prime} 46^{\prime \prime}$ & $107^{\circ} 34^{\prime} 27^{\prime \prime}$ & 11,630 \\
\hline UA26 & North Fork Animas River above West Fork & $37^{\circ} 55^{\prime} 57^{\prime \prime}$ & $107^{\circ} 34^{\prime} 08^{\prime \prime}$ & 11,200 \\
\hline UA27 & North Fork below Burrows Gulch & $37^{\circ} 56^{\prime} 43^{\prime \prime}$ & $107^{\circ} 34^{\prime} 26^{\prime \prime}$ & 11,600 \\
\hline UA28 & North Fork Minnie Gulch & $37^{\circ} 51^{\prime} 44^{\prime \prime}$ & $107^{\circ} 32^{\prime} 48^{\prime \prime}$ & 11,720 \\
\hline UA29 & Picayne Gulch near mouth & $37^{\circ} 54^{\prime} 42^{\prime \prime}$ & $107^{\circ} 33^{\prime} 21^{\prime \prime}$ & 10,640 \\
\hline UA30 & Placer Gulch above Gold Prince Mine & $37^{\circ} 54^{\prime} 30^{\prime \prime}$ & $107^{\circ} 36^{\prime} 06^{\prime \prime}$ & 12,280 \\
\hline UA31 & Placer Gulch above Sunbank Mine & $37^{\circ} 54^{\prime} 56^{\prime \prime}$ & $107^{\circ} 35^{\prime} 29^{\prime \prime}$ & 11,660 \\
\hline UA32 & Placer Gulch below Gold Prince Mine & $37^{\circ} 54^{\prime} 35^{\prime \prime}$ & $107^{\circ} 35^{\prime} 57^{\prime \prime}$ & 12,120 \\
\hline UA33 & Placer Gulch below Sunbank Mine & $37^{\circ} 55^{\prime} 02^{\prime \prime}$ & $107^{\circ} 35^{\prime} 26^{\prime \prime}$ & 11,610 \\
\hline UA34 & South Fork Eureka Gulch & $37^{\circ} 52^{\prime} 54^{\prime \prime}$ & $107^{\circ} 35^{\prime} 37^{\prime \prime}$ & 10,600 \\
\hline UA35 & Spring below London Mine Dump & $37^{\circ} 56^{\prime} 53^{\prime \prime}$ & $107^{\circ} 35^{\prime} 00^{\prime \prime}$ & 11,950 \\
\hline UA36 & Spring in Burns Gulch & $37^{\circ} 54^{\prime} 08^{\prime \prime}$ & $107^{\circ} 32^{\prime} 43^{\prime \prime}$ & 11,400 \\
\hline UA37 & Spring in California Gulch & $37^{\circ} 55^{\prime} 09^{\prime \prime}$ & $107^{\circ} 36^{\prime} 40^{\prime \prime}$ & 12,190 \\
\hline UA38 & Spring in Placer Gulch & $37^{\circ} 54^{\prime} 32^{\prime \prime}$ & $107^{\circ} 35^{\prime} 47^{\prime \prime}$ & 12,090 \\
\hline
\end{tabular}


Table 3. Water-quality sampling sites at selected springs and streams in the upper Animas River watershed (grouped according to three major subbasins of the watershed)

[UA, upper Animas River above Silverton; CC, Cement Creek; MC, Mineral Creek; site locations are shown on plate 1]

\begin{tabular}{|c|c|c|c|c|}
\hline $\begin{array}{c}\text { Site } \\
\text { number }\end{array}$ & Site name & Latitude & Longitude & $\begin{array}{c}\text { Elevation } \\
\text { (feet) }\end{array}$ \\
\hline UA39 & Spring in South Fork Eureka Gulch & $37^{\circ} 52^{\prime} 50^{\prime \prime}$ & $107^{\circ} 36^{\prime} 03^{\prime \prime}$ & 11,300 \\
\hline UA40 & Spring near Houghton Mountain & $37^{\circ} 56^{\prime} 47^{\prime \prime}$ & $107^{\circ} 35^{\prime} 09^{\prime \prime}$ & 11,840 \\
\hline UA41 & Spring near Silver Wing Mine & $37^{\circ} 54^{\prime} 12^{\prime \prime}$ & $107^{\circ} 33^{\prime} 20^{\prime \prime}$ & 10,360 \\
\hline UA42 & Stream below Denver Lake & $37^{\circ} 57^{\prime} 00^{\prime \prime}$ & $107^{\circ} 34^{\prime} 21^{\prime \prime}$ & 11,750 \\
\hline UA43 & Stream below Lucky Jack Mine & $37^{\circ} 57^{\prime} 10^{\prime \prime}$ & $107^{\circ} 34^{\prime} 21^{\prime \prime}$ & 11,760 \\
\hline UA44 & Stream in Placer Gulch & $37^{\circ} 54^{\prime} 39^{\prime \prime}$ & $107^{\circ} 36^{\prime} 03^{\prime \prime}$ & 12,220 \\
\hline UA45 & Upper Animas River above Cinnamon Creek & $37^{\circ} 55^{\prime} 31^{\prime \prime}$ & $107^{\circ} 33^{\prime} 50^{\prime \prime}$ & 10,850 \\
\hline UA46 & Upper Animas River above Silver Wing Mine & $37^{\circ} 54^{\prime} 15^{\prime \prime}$ & $107^{\circ} 33^{\prime} 21^{\prime \prime}$ & 10,380 \\
\hline UA47 & Upper Burns Gulch & $37^{\circ} 54^{\prime} 04^{\prime \prime}$ & $107^{\circ} 32^{\prime} 35^{\prime \prime}$ & 11,600 \\
\hline UA48 & Burns Gulch & $37^{\circ} 54^{\prime} 18^{\prime \prime}$ & $107^{\circ} 32^{\prime} 55^{\prime \prime}$ & 11,240 \\
\hline UA49 & Upper Picayne Creek & $37^{\circ} \quad 54^{\prime} 34^{\prime \prime}$ & $107^{\circ} 34^{\prime} 32^{\prime \prime}$ & 11,830 \\
\hline UA50 & West Fork Animas River below Placer Gulch & $37^{\circ} 55^{\prime} 51^{\prime \prime}$ & $107^{\circ} 34^{\prime} 55^{\prime \prime}$ & 11,370 \\
\hline UA51 & West Fork Animas River near Animas Forks & $37^{\circ} 55^{\prime} 53^{\prime \prime}$ & $107^{\circ} 34^{\prime} 13^{\prime \prime}$ & 11,120 \\
\hline $\mathrm{CCl}$ & Bogwan Spring & $37^{\circ} 52^{\prime} 45^{\prime \prime}$ & $107^{\circ} 40^{\prime} 11^{\prime \prime}$ & 10,265 \\
\hline $\mathrm{CC} 2$ & Cascade Gulch near mouth & $37^{\circ} 51^{\prime} 56^{\prime \prime}$ & $107^{\circ} 40^{\prime} 26^{\prime \prime}$ & 10,100 \\
\hline $\mathrm{CC} 3$ & Cement Creek above Mogul Mine & $37^{\circ} 54^{\prime} 37^{\prime \prime}$ & $107^{\circ} 38^{\prime} 23^{\prime \prime}$ & 11,360 \\
\hline $\mathrm{CC} 4$ & Cement Creek below Ross Basin & $37^{\circ} 54^{\prime} 34^{\prime \prime}$ & $107^{\circ} 37^{\prime} 49^{\prime \prime}$ & 11,760 \\
\hline $\mathrm{CC} 5$ & Dry Gulch above Evelyn Mine & $37^{\circ} 53^{\prime} 19^{\prime \prime}$ & $107^{\circ} 39^{\prime} 51^{\prime \prime}$ & 10,500 \\
\hline $\mathrm{CC} 6$ & East Queen Anne Creek & $37^{\circ} 54^{\prime} 48^{\prime \prime}$ & $107^{\circ} 37^{\prime} 48^{\prime \prime}$ & 12,200 \\
\hline $\mathrm{CC} 7$ & Fairview Gulch near mouth & $37^{\circ} 52^{\prime} 23^{\prime \prime}$ & $107^{\circ} 40^{\prime} 38^{\prime \prime}$ & 10,540 \\
\hline $\mathrm{CC} 8$ & Georgia Gulch near mouth & $37^{\circ} 52^{\prime} 42^{\prime \prime}$ & $107^{\circ} 40^{\prime} 21^{\prime \prime}$ & 10,400 \\
\hline $\mathrm{CC} 9$ & Hancock Gulch near mouth & $37^{\circ} 50^{\prime} 08^{\prime \prime}$ & $107^{\circ} 40^{\prime} 34^{\prime \prime}$ & 9,720 \\
\hline $\mathrm{CC} 10$ & Stream south of Hancock Gulch above adit & $37^{\circ} 50^{\prime} 12^{\prime \prime}$ & $107^{\circ} 39^{\prime} 46^{\prime \prime}$ & 10,440 \\
\hline $\mathrm{CC} 11$ & Stream south of Hancock Gulch near mouth & $37^{\circ} 49^{\prime} 46^{\prime \prime}$ & $107^{\circ} 40^{\prime} 10^{\prime \prime}$ & 9,649 \\
\hline $\mathrm{CC} 12$ & Illinois Gulch above Yukon Mine & $37^{\circ} 51^{\prime} 01^{\prime \prime}$ & $107^{\circ} 40^{\prime} 29^{\prime \prime}$ & 9,960 \\
\hline $\mathrm{CC} 13$ & Middle Fork Cement Creek near mouth & $37^{\circ} 53^{\prime} 08^{\prime \prime}$ & $107^{\circ} 38^{\prime} 50^{\prime \prime}$ & 10,710 \\
\hline $\mathrm{CC} 14$ & Minnesota Gulch near mouth & $37^{\circ} 51^{\prime} 47^{\prime \prime}$ & $107^{\circ} 40^{\prime} 33^{\prime \prime}$ & 10,800 \\
\hline $\mathrm{CC} 15$ & Minnehaha near mouth & $37^{\circ} 53^{\prime} 14^{\prime \prime}$ & $107^{\circ} 38^{\prime} 49^{\prime \prime}$ & 10,640 \\
\hline $\mathrm{CC} 16$ & Niagara Gulch below Irene Mine & $37^{\circ} 50^{\prime} 28^{\prime \prime}$ & $107^{\circ} 42^{\prime} 04^{\prime \prime}$ & 11,600 \\
\hline $\mathrm{CC} 17$ & Niagara Gulch near mouth & $37^{\circ} 50^{\prime} 23^{\prime \prime}$ & $107^{\circ} 40^{\prime} 47^{\prime \prime}$ & 9,800 \\
\hline $\mathrm{CC} 18$ & North Fork Cement Creek above upper Gold King Mine & $37^{\circ} 53^{\prime} 44^{\prime \prime}$ & $107^{\circ} 37^{\prime} 49^{\prime \prime}$ & 12,090 \\
\hline $\mathrm{CC} 19$ & North Fork Cement Creek above upper Gold King Mine & $37^{\circ} 53^{\prime} 38^{\prime \prime}$ & $107^{\circ} 38^{\prime} 15^{\prime \prime}$ & 11,430 \\
\hline $\mathrm{CC} 20$ & Porcupine Gulch above mines & $37^{\circ} 51^{\prime} 35^{\prime \prime}$ & $107^{\circ} 41^{\prime} 03^{\prime \prime}$ & 10,920 \\
\hline $\mathrm{CC} 21$ & Porcupine Gulch near mouth & $37^{\circ} 51^{\prime} 31^{\prime \prime}$ & $107^{\circ} 40^{\prime} 35^{\prime \prime}$ & 10,080 \\
\hline $\mathrm{CC} 22$ & Prospect Gulch above mouth & $37^{\circ} 53^{\prime} 04^{\prime \prime}$ & $107^{\circ} 40^{\prime} 23^{\prime \prime}$ & 10,600 \\
\hline $\mathrm{CC} 23$ & Prospect Gulch above Red Chemotroph Spring & $37^{\circ} 53^{\prime} 00^{\prime \prime}$ & $107^{\circ} 40^{\prime} 17^{\prime \prime}$ & 10,470 \\
\hline $\mathrm{CC} 24$ & Prospect Gulch below Henrietta Mine & $37^{\circ} 53^{\prime} 20^{\prime \prime}$ & $107^{\circ} 40^{\prime} 37^{\prime \prime}$ & 10,920 \\
\hline $\mathrm{CC} 25$ & Prospect Gulch below Red Chemotroph Spring & $37^{\circ} 52^{\prime} 59^{\prime \prime}$ & $107^{\circ} 40^{\prime} 03^{\prime \prime}$ & 10,440 \\
\hline
\end{tabular}


Table 3. Water-quality sampling sites at selected springs and streams in the upper Animas River watershed (grouped according to three major subbasins of the watershed)

[UA, upper Animas River above Silverton; CC, Cement Creek; MC, Mineral Creek; site locations are shown on plate 1]

\begin{tabular}{|c|c|c|c|c|}
\hline $\begin{array}{c}\text { Site } \\
\text { number }\end{array}$ & Site name & Latitude & Longitude & $\begin{array}{l}\text { Elevation } \\
\text { (feet) }\end{array}$ \\
\hline $\mathrm{CC} 26$ & Prospect Gulch near mouth & $37^{\circ} 52^{\prime} 58^{\prime \prime}$ & $107^{\circ} 40^{\prime} 02^{\prime \prime}$ & 10,360 \\
\hline $\mathrm{CC} 27$ & Queen Anne Creek above Queen Anne Mine & $37^{\circ} 54^{\prime} 51^{\prime \prime}$ & $107^{\circ} 37^{\prime} 47^{\prime \prime}$ & 12,200 \\
\hline $\mathrm{CC} 28$ & Queen Anne Creek near mouth & $37^{\circ} 54^{\prime} 37^{\prime \prime}$ & $107^{\circ} 37^{\prime} 56^{\prime \prime}$ & 11,720 \\
\hline CC29 & Red Chemotroph Spring & $37^{\circ} 53^{\prime} 00^{\prime \prime}$ & $107^{\circ} 40^{\prime} 14^{\prime \prime}$ & 10,450 \\
\hline CC30 & South Fork Cement Creek above Silver Ledge Mine & $37^{\circ} 52^{\prime} 33^{\prime \prime}$ & $107^{\circ} 38^{\prime} 38^{\prime \prime}$ & 10,974 \\
\hline $\mathrm{CC} 31$ & South Fork Cement Creek below Big Colorado Mine & $37^{\circ} 52^{\prime} 41^{\prime \prime}$ & $107^{\circ} 38^{\prime} 42^{\prime \prime}$ & 10,912 \\
\hline $\mathrm{CC} 32$ & South Fork Cement Creek below Velocity Lake & $37^{\circ} 52^{\prime} 08^{\prime \prime}$ & $107^{\circ} 38^{\prime} 37^{\prime \prime}$ & 11,305 \\
\hline CC33 & South Fork Cement Creek at Gladstone & $37^{\circ} 533^{\prime \prime \prime}$ & $107^{\circ} 39^{\prime} 10^{\prime \prime}$ & 10,469 \\
\hline $\mathrm{CC} 34$ & Spring near Big Colorado Mine & $37^{\circ} 52^{\prime} 36^{\prime \prime}$ & $107^{\circ} 38^{\prime} 40^{\prime \prime}$ & 10,950 \\
\hline CC35 & Spring below Red Mountain \#3 & $37^{\circ} 53^{\prime} 53^{\prime \prime}$ & $107^{\circ} 41^{\prime} 08^{\prime \prime}$ & 12,120 \\
\hline $\mathrm{CC} 36$ & Spring below Red Mountain \#3 & $37^{\circ} 53^{\prime} 54^{\prime \prime}$ & $107^{\circ} 41^{\prime} 08^{\prime \prime}$ & 12,120 \\
\hline CC37 & Spring in Cement Creek & $37^{\circ} 53^{\prime} 18^{\prime \prime}$ & $107^{\circ} 39^{\prime} 41^{\prime \prime}$ & 10,436 \\
\hline CC38 & Spring in Dry Gulch & $37^{\circ} 54^{\prime} 01^{\prime \prime}$ & $107^{\circ} 40^{\prime} 25^{\prime \prime}$ & 11,690 \\
\hline CC39 & Spring in Prospect Gulch & $37^{\circ} 53 ' 23^{\prime \prime}$ & $107^{\circ} 40^{\prime} 20^{\prime \prime}$ & 11,120 \\
\hline $\mathrm{CC} 40$ & Spring in Middle Fork Cement Creek below mine & $37^{\circ} 52^{\prime} 53^{\prime \prime}$ & $107^{\circ} 38^{\prime} 15^{\prime \prime}$ & 11,400 \\
\hline $\mathrm{CC} 41$ & Spring in Minnehaha Basin & $37^{\circ} 53^{\prime} 20^{\prime \prime}$ & $107^{\circ} 37^{\prime} 57^{\prime \prime}$ & 11,780 \\
\hline $\mathrm{CC} 42$ & Spring in Niagara Gulch & $37^{\circ} 50^{\prime} 28^{\prime \prime}$ & $107^{\circ} 42^{\prime} 11^{\prime \prime}$ & 11,720 \\
\hline $\mathrm{CC} 43$ & Spring in Prospect Gulch & $37^{\circ} 53^{\prime} 21^{\prime \prime}$ & $107^{\circ} 41^{\prime} 31^{\prime \prime}$ & 12,070 \\
\hline $\mathrm{CC} 44$ & Spring in Prospect Gulch & $37^{\circ} 53^{\prime} 21^{\prime \prime}$ & $107^{\circ} 41^{\prime} 25^{\prime \prime}$ & 12,020 \\
\hline $\mathrm{CC} 45$ & Spring in Prospect Gulch & $37^{\circ} 53^{\prime} 43^{\prime \prime}$ & $107^{\circ} 41^{\prime 22 "}$ & 11,860 \\
\hline CC46 & Spring in Prospect Gulch & $37^{\circ} 53^{\prime} 48^{\prime \prime}$ & $107^{\circ} 40^{\prime} 39^{\prime \prime}$ & 11,915 \\
\hline $\mathrm{CC} 47$ & Spring in Prospect Gulch & $37^{\circ} 53^{\prime} 46^{\prime \prime}$ & $107^{\circ} 40^{\prime} 57^{\prime \prime}$ & 11,910 \\
\hline $\mathrm{CC} 48$ & Spring in Prospect Gulch & $37^{\circ} 53^{\prime} 36^{\prime \prime}$ & $107^{\circ} 41^{\prime} 15^{\prime \prime}$ & 11,730 \\
\hline CC49 & Spring in Prospect Gulch & $37^{\circ} 53^{\prime} 25^{\prime \prime}$ & $107^{\circ} 41^{\prime} 34^{\prime \prime}$ & 12,050 \\
\hline CC50 & Spring in Ross Basin & $37^{\circ} 54^{\prime} 13^{\prime \prime}$ & $107^{\circ} 37^{\prime} 32^{\prime \prime}$ & 12,240 \\
\hline CC51 & Spring in Ross Basin & $37^{\circ} 54^{\prime} 15^{\prime \prime}$ & $107^{\circ} 37^{\prime} 43^{\prime \prime}$ & 12,200 \\
\hline CC52 & Spring in South Fork Cement Creek & $37^{\circ} 53^{\prime} 02^{\prime \prime}$ & $107^{\circ} 38^{\prime} 46^{\prime \prime}$ & 10,695 \\
\hline CC53 & Spring near Corkscrew Pass & $37^{\circ} 54^{\prime} 12^{\prime \prime}$ & $107^{\circ} 39^{\prime} 32^{\prime \prime}$ & 11,840 \\
\hline CC54 & Spring near Dry Gulch & $37^{\circ} 53^{\prime} 18^{\prime \prime}$ & $107^{\circ} 39^{\prime} 39^{\prime \prime}$ & 10,390 \\
\hline CC55 & Spring near Gladstone & $37^{\circ} 53^{\prime} 22^{\prime \prime}$ & $107^{\circ} 39^{\prime} 20^{\prime \prime}$ & 10,400 \\
\hline CC56 & Spring near Queene Anne Creek & $37^{\circ} 54^{\prime} 48^{\prime \prime}$ & $107^{\circ} 37^{\prime} 43^{\prime \prime}$ & 12,200 \\
\hline CC57 & Stream below Henrietta \#3 Mine & $37^{\circ} 53^{\prime} 20^{\prime \prime}$ & $107^{\circ} 40^{\prime} 40^{\prime \prime}$ & 11,000 \\
\hline CC58 & Stream below Kansas City Mine & $37^{\circ} 52^{\prime} 55^{\prime \prime}$ & $107^{\circ} 40^{\prime} 59^{\prime \prime}$ & 11,400 \\
\hline CC59 & Stream below Lead Carbonate Mill & $37^{\circ} 53^{\prime} 24^{\prime \prime}$ & $107^{\circ} 37^{\prime} 59^{\prime \prime}$ & 11,750 \\
\hline CC60 & Stream in Middle Fork Cement Creek & $37^{\circ} 52^{\prime} 51^{\prime \prime}$ & $107^{\circ} 38^{\prime} 09^{\prime \prime}$ & 11,570 \\
\hline CC61 & Stream in Minnesota Gulch & $37^{\circ} 52^{\prime} 03^{\prime \prime}$ & $107^{\circ} 41^{\prime} 08^{\prime \prime}$ & 10,840 \\
\hline CC62 & Stream in Minnesota Gulch & $37^{\circ} 52^{\prime} 02^{\prime \prime}$ & $107^{\circ} 41^{\prime} 00^{\prime \prime}$ & 10,680 \\
\hline CC63 & Stream in Niagara Gulch & $37^{\circ} 50^{\prime} 28^{\prime \prime}$ & $107^{\circ} 41^{\prime} 50^{\prime \prime}$ & 11,240 \\
\hline CC64 & Stream in Niagara Gulch & $37^{\circ} 50^{\prime} 32^{\prime \prime}$ & $107^{\circ} 41^{\prime} 48^{\prime \prime}$ & 11,300 \\
\hline
\end{tabular}


Table 3. Water-quality sampling sites at selected springs and streams in the upper Animas River watershed (grouped according to three major subbasins of the watershed) .

[UA, upper Animas River above Silverton; CC, Cement Creek; MC, Mineral Creek; site locations are shown on plate 1]

\begin{tabular}{|c|c|c|c|c|}
\hline $\begin{array}{c}\text { Site } \\
\text { number }\end{array}$ & Site name & Latitude & Longitude & $\begin{array}{c}\text { Elevation } \\
\text { (feet) }\end{array}$ \\
\hline CC65 & Stream in Porcupine Gulch & $37^{\circ} 51^{\prime} 40^{\prime \prime}$ & $107^{\circ} 41^{\prime} 03^{\prime \prime}$ & 11,000 \\
\hline CC66 & Stream in Prospect Gulch & $37^{\circ} 53^{\prime} 37^{\prime \prime}$ & $107^{\circ} 41^{\prime} 20^{\prime \prime}$ & 11,920 \\
\hline $\mathrm{CC} 67$ & Stream in Prospect Gulch & $37^{\circ} 53^{\prime} 35^{\prime \prime}$ & $107^{\circ} 41^{\prime} 02^{\prime \prime}$ & 11,320 \\
\hline CC68 & Stream in Prospect Gulch & $37^{\circ} 53^{\prime} 29^{\prime \prime}$ & $107^{\circ} 41^{\prime} 10^{\prime \prime}$ & 11,550 \\
\hline CC69 & Stream in Prospect Gulch & $37^{\circ} 53^{\prime} 28^{\prime \prime}$ & $107^{\circ} 41^{\prime} 11^{\prime \prime}$ & 11,550 \\
\hline $\mathrm{CC} 70$ & Stream in Prospect Gulch & $37^{\circ} 53^{\prime} 30^{\prime \prime}$ & $107^{\circ} 41^{\prime} 21^{\prime \prime}$ & 11,700 \\
\hline $\mathrm{CC} 71$ & Stream in Prospect Gulch & $37^{\circ} 53^{\prime} 28^{\prime \prime}$ & $107^{\circ} 41^{\prime} 13^{\prime \prime}$ & 11,570 \\
\hline $\mathrm{CC} 72$ & Stream in Prospect Gulch & $37^{\circ} 53^{\prime} 35^{\prime \prime}$ & $107^{\circ} 41^{\prime} 19^{\prime \prime}$ & 11,720 \\
\hline $\mathrm{CC} 73$ & Stream in Prospect Gulch & $37^{\circ} 53^{\prime} 37^{\prime \prime}$ & $107^{\circ} 41^{\prime} 31^{\prime \prime}$ & 11,960 \\
\hline $\mathrm{CC} 74$ & Stream in Prospect Gulch & $37^{\circ} 53^{\prime} 30^{\prime \prime}$ & $107^{\circ} 41^{\prime} 34^{\prime \prime}$ & 11,960 \\
\hline $\mathrm{CC} 75$ & Stream in Prospect Gulch & $37^{\circ} 53^{\prime} 24^{\prime \prime}$ & $107^{\circ} 41^{\prime} 28^{\prime \prime}$ & 11,850 \\
\hline $\mathrm{CC} 76$ & Stream in Prospect Gulch & $37^{\circ} 53^{\prime} 29^{\prime \prime}$ & $107^{\circ} 41^{\prime} 23^{\prime \prime}$ & 11,820 \\
\hline $\mathrm{CC} 77$ & Stream in Prospect Gulch & $37^{\circ} 53^{\prime} 31^{\prime \prime}$ & $107^{\circ} 41^{\prime} 22^{\prime \prime}$ & 11,740 \\
\hline $\mathrm{CC} 78$ & Stream near Adams Mine & $37^{\circ} 54^{\prime} 00^{\prime \prime}$ & $107^{\circ} 38^{\prime} 29^{\prime \prime}$ & 11,200 \\
\hline CC79 & Telephone Gulch above adit & $37^{\circ} 52^{\prime} 24^{\prime \prime}$ & $107^{\circ} 40^{\prime} 06^{\prime \prime}$ & 10,560 \\
\hline $\mathrm{CC} 80$ & Telephone Gulch at mouth & $37^{\circ} 52^{\prime} 25^{\prime \prime}$ & $107^{\circ} 40^{\prime} 17^{\prime \prime}$ & 10,220 \\
\hline $\mathrm{CC} 81$ & Tirbutary of upper Cement Creek & $37^{\circ} 54^{\prime} 49^{\prime \prime}$ & $107^{\circ} 38^{\prime} 26^{\prime \prime}$ & 11,900 \\
\hline $\mathrm{CC} 82$ & Topeka Gulch near mouth & $37^{\circ} 50^{\prime} 46^{\prime \prime}$ & $107^{\circ} 40^{\prime} 43^{\prime \prime}$ & 9,895 \\
\hline $\mathrm{CC} 83$ & Tributary of Queen Anne Creek & $37^{\circ} 54^{\prime} 51^{\prime \prime}$ & $107^{\circ} 37^{\prime} 41^{\prime \prime}$ & 12,220 \\
\hline $\mathrm{CC} 84$ & Tributary of upper Cement Creek & $37^{\circ} 54^{\prime} 50^{\prime \prime}$ & $107^{\circ} 38^{\prime} 13^{\prime \prime}$ & 12,080 \\
\hline $\mathrm{CC} 85$ & Upper Cement Creek below upper Ross Basin & $37^{\circ} 54^{\prime} 32^{\prime \prime}$ & $107^{\circ} 37^{\prime} 41^{\prime \prime}$ & 12,000 \\
\hline $\mathrm{CC} 86$ & Upper Georgia Gulch & $37^{\circ} 53^{\prime} 03^{\prime \prime}$ & $107^{\circ} 41^{\prime} 18^{\prime \prime}$ & 11,840 \\
\hline $\mathrm{MCl}$ & Bear Creek near mouth & $37^{\circ} 48^{\prime} 46^{\prime \prime}$ & $107^{\circ} 41^{\prime} 54^{\prime \prime}$ & 9,560 \\
\hline $\mathrm{MC2}$ & Browns Gulch above Brooklyn Mine & $37^{\circ} 51^{\prime} 37^{\prime \prime}$ & $107^{\circ} 42^{\prime} 35^{\prime \prime}$ & 11,200 \\
\hline $\mathrm{MC3}$ & Browns Gulch near mouth & $37^{\circ} 51^{\prime} 24^{\prime \prime}$ & $107^{\circ} 43^{\prime} 23^{\prime \prime}$ & 10,260 \\
\hline MC4 & Browns Gulch below Brooklyn Mine & $37^{\circ} 51^{\prime 2} 26^{\prime \prime}$ & $107^{\circ} 42^{\prime} 56^{\prime \prime}$ & 10,800 \\
\hline MC5 & Crystal Creek near mouth & $37^{\circ} 50^{\prime} 33^{\prime \prime}$ & $107^{\circ} 45^{\prime} 51^{\prime \prime}$ & 10,640 \\
\hline MC6 & Iron bog in South Fork Mineral Creek & $37^{\circ} 48^{\prime} 58^{\prime \prime}$ & $107^{\circ} 43^{\prime} 49^{\prime \prime}$ & 9,590 \\
\hline $\mathrm{MC7}$ & Middle Fork Mineral Creek above Paradise Portal & $37^{\circ} 50^{\prime} 27^{\prime \prime}$ & $107^{\circ} 45^{\prime} 56^{\prime \prime}$ & 10,680 \\
\hline MC8 & Middle Fork Mineral Creek below Paradise Portal & $37^{\circ} 50^{\prime} 33^{\prime \prime}$ & $107^{\circ} 45^{\prime} 50^{\prime \prime}$ & 10,660 \\
\hline MC9 & Mill Creek near mouth & $37^{\circ} 52^{\prime} 21^{\prime \prime}$ & $107^{\circ} 44^{\prime} 08^{\prime \prime}$ & 10,500 \\
\hline $\mathrm{MC10}$ & Mineral Creek near Red Mountain Pass & $37^{\circ} 53^{\prime} 32^{\prime \prime}$ & $107^{\circ} 43^{\prime} 00^{\prime \prime}$ & 10,980 \\
\hline MC11 & Moss bog in South Fork Mineral Creek & $37^{\circ} 48^{\prime} 50^{\prime \prime}$ & $107^{\circ} 44^{\prime} 16^{\prime \prime}$ & 9,662 \\
\hline $\mathrm{MC12}$ & North tributary to Bighorn Gulch above mines & $37^{\circ} 53^{\prime} 16^{\prime \prime}$ & $107^{\circ} 42^{\prime} 29^{\prime \prime}$ & 11,440 \\
\hline MC13 & North tributary to Bighorn Gulch below mines & $37^{\circ} 53^{\prime} 13^{\prime \prime}$ & $107^{\circ} 42^{\prime} 36^{\prime \prime}$ & 11,290 \\
\hline MC14 & Porphyry Gulch above Bullion King Mine & $37^{\circ} 53^{\prime} 16^{\prime \prime}$ & $107^{\circ} 44^{\prime} 28^{\prime \prime}$ & 12,340 \\
\hline MC15 & Porphyry Gulch below Bullion King Mine & $37^{\circ} 53^{\prime} 17^{\prime \prime}$ & $107^{\circ} 44^{\prime} 27^{\prime \prime}$ & 12,000 \\
\hline $\mathrm{MC16}$ & Porphyry Gulch below Highway 550 & $37^{\circ} 53^{\prime} 05^{\prime \prime}$ & $107^{\circ} 43^{\prime} 20^{\prime \prime}$ & 10,780 \\
\hline MC17 & Red Tributary of Middle Fork Mineral Creek & $37^{\circ} 50^{\prime} 25^{\prime \prime}$ & $107^{\circ} 44^{\prime} 57^{\prime \prime}$ & 10,460 \\
\hline
\end{tabular}


Table 3. Water-quality sampling sites at selected springs and streams in the upper Animas River watershed (grouped according to three major subbasins of the watershed)

[UA, upper Animas River above Silverton; CC, Cement Creek; MC, Mineral Creek; site locations are shown on plate 1]

\begin{tabular}{|c|c|c|c|c|}
\hline $\begin{array}{c}\text { Site } \\
\text { number }\end{array}$ & Site name & Latitude & Longitude & $\begin{array}{c}\text { Elevation } \\
\text { (feet) }\end{array}$ \\
\hline MC18 & Red Tributary of Middle Fork Mineral Creek & $37^{\circ} 50^{\prime} 23^{\prime \prime}$ & $107^{\circ} 44^{\prime} 59^{\prime \prime}$ & 10,500 \\
\hline MC19 & Red Tributary of Middle Fork Mineral Creek & $37^{\circ} 50^{\prime} 15^{\prime \prime}$ & $107^{\circ} 45^{\prime} 08^{\prime \prime}$ & 10,700 \\
\hline MC20 & Red Tributary of Middle Fork Mineral Creek & $37^{\circ} 50^{\prime} 13^{\prime \prime}$ & $107^{\circ} 45^{\prime} 11^{\prime \prime}$ & 10,750 \\
\hline MC21 & Red Tributary of Middle Fork Mineral Creek & $37^{\circ} 49^{\prime} 55^{\prime \prime}$ & $107^{\circ} 45^{\prime} 34^{\prime \prime}$ & 11,360 \\
\hline MC22 & Red Tributary of Middle Fork Mineral Creek & $37^{\circ} 50^{\prime} 38^{\prime \prime}$ & $107^{\circ} 44^{\prime} 49^{\prime \prime}$ & 10,360 \\
\hline MC23 & Spring in Red Tributary of Middle Fork Mineral Creek & $37^{\circ} 50^{\prime} 14^{\prime \prime}$ & $107^{\circ} 45^{\prime} 10^{\prime \prime}$ & 10,720 \\
\hline MC24 & Spring in Red Tributary of Middle Fork Mineral Creek & $37^{\circ} 50^{\prime} 17^{\prime \prime}$ & $107^{\circ} 44^{\prime} 52^{\prime \prime}$ & 10,800 \\
\hline MC25 & Spring in Red Tributary of Middle Fork Mineral Creek & $37^{\circ} 50^{\prime} 15^{\prime \prime}$ & $107^{\circ} 45^{\prime} 03^{\prime \prime}$ & 10,720 \\
\hline MC26 & Spring in Red Tributary of Middle Fork Mineral Creek & $37^{\circ} 49^{\prime} 59^{\prime \prime}$ & $107^{\circ} 45^{\prime} 30^{\prime \prime}$ & 11,300 \\
\hline MC27 & Spring in Red Tributary of Middle Fork Mineral Creek & $37^{\circ} 49^{\prime} 43^{\prime \prime}$ & $107^{\circ} 45^{\prime} 37^{\prime \prime}$ & 11,660 \\
\hline MC28 & Spring in Battleship Slide & $37^{\circ} 50^{\prime} 01^{\prime \prime}$ & $107^{\circ} 43^{\prime} 49^{\prime \prime}$ & 10,040 \\
\hline MC29 & Spring in Battleship Slide & $37^{\circ} 50^{\prime} 00^{\prime \prime}$ & $107^{\circ} 43^{\prime} 54^{\prime \prime}$ & 10,180 \\
\hline MC30 & Spring in Battleship Slide & $37^{\circ} 50^{\prime} 00^{\prime \prime}$ & $107^{\circ} 43^{\prime} 51^{\prime \prime}$ & 10,200 \\
\hline MC31 & Spring near Burro Bridge & $37^{\circ} 51^{\prime} 06^{\prime \prime}$ & $107^{\circ} 43^{\prime} 30^{\prime \prime}$ & 10,040 \\
\hline MC32 & Spring near Chattanooga & $37^{\circ} 52^{\prime} 15^{\prime \prime}$ & $107^{\circ} 43^{\prime} 34^{\prime \prime}$ & 10,280 \\
\hline MC33 & Spring near Chattanooga & $37^{\circ} 52^{\prime} 14^{\prime \prime}$ & $107^{\circ} 43^{\prime} 45^{\prime \prime}$ & 10,600 \\
\hline MC34 & Spring near Mount Moly & $37^{\circ} 49^{\prime} 18^{\prime \prime}$ & $107^{\circ} 44^{\prime} 10^{\prime \prime}$ & 10,690 \\
\hline MC35 & Spring near Paradise Portal & $37^{\circ} 50^{\prime} 35^{\prime \prime}$ & $107^{\circ} 45^{\prime} 42^{\prime \prime}$ & 10,640 \\
\hline MC36 & Spring northwest of Burro Bridge & $37^{\circ} 51^{\prime} 20^{\prime \prime}$ & $107^{\circ} 43^{\prime} 52^{\prime \prime}$ & 10,760 \\
\hline MC37 & Spring northwest of Chattanooga beaver bogs & $37^{\circ} 52^{\prime} 16^{\prime \prime}$ & $107^{\circ} 43^{\prime} 38^{\prime \prime}$ & 10,310 \\
\hline MC38 & Spring southwest of Ohio Peak & $37^{\circ} 50^{\prime} 58^{\prime \prime}$ & $107^{\circ} 42^{\prime} 06^{\prime \prime}$ & 11,840 \\
\hline MC39 & Stream below Congress Mine & $37^{\circ} 53^{\prime} 26^{\prime \prime}$ & $107^{\circ} 42^{\prime} 27^{\prime \prime}$ & 11,430 \\
\hline MC40 & Stream below mine in U S Basin & $37^{\circ} 52^{\prime} 28^{\prime \prime}$ & $107^{\circ} 41^{\prime} 46^{\prime \prime}$ & 12,260 \\
\hline MC41 & Stream in Cemetary Slide & $37^{\circ} 52^{\prime} 14^{\prime \prime}$ & $107^{\circ} 43^{\prime} 13^{\prime \prime}$ & 10,420 \\
\hline MC42 & Stream in Cemetary Slide & $37^{\circ} 52^{\prime} 14^{\prime \prime}$ & $107^{\circ} 43^{\prime} 07^{\prime \prime}$ & 10,620 \\
\hline MC43 & Stream in Red Tributary of Middle Fork Mineral Creek & $37^{\circ} 50^{\prime} 23^{\prime \prime}$ & $107^{\circ} 44^{\prime} 58^{\prime \prime}$ & 10,500 \\
\hline MC44 & Stream in Red Tributary of Middle Fork Mineral Creek & $37^{\circ} 50^{\prime} 13^{\prime \prime}$ & $107^{\circ} 45^{\prime} 09^{\prime \prime}$ & 10,760 \\
\hline MC45 & Stream in Battleship Slide & $37^{\circ} 50^{\prime} 05^{\prime \prime}$ & $107^{\circ} 43^{\prime} 39^{\prime \prime}$ & 9,730 \\
\hline MC46 & Stream in Cemetary Slide & $37^{\circ} 52^{\prime} 14^{\prime \prime}$ & $107^{\circ} 43^{\prime} 01^{\prime \prime}$ & 10,680 \\
\hline MC47 & Stream near Mount Moly & $37^{\circ} 49^{\prime} 02^{\prime \prime}$ & $107^{\circ} 44^{\prime} 29^{\prime \prime}$ & 10,050 \\
\hline MC48 & Stream near Mount Moly & $37^{\circ} 50^{\prime} 14^{\prime \prime}$ & $107^{\circ} 43^{\prime} 39^{\prime \prime}$ & 9,740 \\
\hline MC49 & Stream near Mount Moly & $37^{\circ} 48^{\prime} 51^{\prime \prime}$ & $107^{\circ} 44^{\prime} 28^{\prime \prime}$ & 9,680 \\
\hline MC50 & Stream south of Browns Gulch & $37^{\circ} 50^{\prime} 51^{\prime \prime}$ & $107^{\circ} 43^{\prime} 25^{\prime \prime}$ & 10,110 \\
\hline MC51 & Stream south of Browns Gulch & $37^{\circ} 50^{\prime} 48^{\prime \prime}$ & $107^{\circ} 43^{\prime} 15^{\prime \prime}$ & 10,200 \\
\hline MC52 & Stream south of Browns Gulch & $37^{\circ} 50^{\prime} 50^{\prime \prime}$ & $107^{\circ} 43^{\prime} 14^{\prime \prime}$ & 10,200 \\
\hline MC53 & Stream southwest of Ohio Peak & $37^{\circ} 50^{\prime} 57^{\prime \prime}$ & $107^{\circ} 42^{\prime} 12^{\prime \prime}$ & 11,800 \\
\hline MC54 & Upper Zuni Gulch & $37^{\circ} 49^{\prime} 50^{\prime \prime}$ & $107^{\circ} 42^{\prime} 17^{\prime \prime}$ & 11,020 \\
\hline MC55 & Upper Zuni Gulch & $37^{\circ} 49^{\prime} 49^{\prime \prime}$ & $107^{\circ} 42^{\prime} 15^{\prime \prime}$ & 11,020 \\
\hline MC56 & Zuni Gulch near mouth & $37^{\circ} 49^{\prime} 09^{\prime \prime}$ & $107^{\circ} 42^{\prime} 24^{\prime \prime}$ & 9,580 \\
\hline MC57 & Spring below mine northwest of Burro Bridge & $37^{\circ} 51^{\prime} 19^{\prime \prime}$ & $107^{\circ} 43^{\prime} 42^{\prime \prime}$ & 10,270 \\
\hline
\end{tabular}


Table 4. Analytical techniques and corresponding detection limits used for water-quality analyses

[ICP-AES, inductively coupled plasma-atomic emission spectroscopy; AA, atomic absorption spectroscopy; $\mathrm{mg} / \mathrm{L}$, milligrams per liter; $\mu \mathrm{g} / \mathrm{L}$, micrograms per liter]

\begin{tabular}{llr}
\hline Constituent & Analytical method & $\begin{array}{c}\text { Detection } \\
\text { limit }\end{array}$ \\
\hline Calcium & ICP-AES & $0.15 \mathrm{mg} / \mathrm{L}$ \\
Magnesium & ICP-AES & $0.01 \mathrm{mg} / \mathrm{L}$ \\
Sodium & ICP-AES & $0.10 \mathrm{mg} / \mathrm{L}$ \\
Potassium & AA & $0.01 \mathrm{mg} / \mathrm{L}$ \\
Alkalinity & Incremental titration & $0.5 \mathrm{mg} / \mathrm{L}$ \\
Sulfate & Turbidimetric method & $1.0 \mathrm{mg} / \mathrm{L}$ \\
Silica & ICP-AES & $0.20 \mathrm{mg} / \mathrm{L}$ \\
Aluminum & ICP-AES & $40 \mu \mathrm{g} / \mathrm{L}$ \\
Barium & ICP-AES & $2 \mu \mathrm{g} / \mathrm{L}$ \\
Beryllium & ICP-AES & $1 \mu \mathrm{g} / \mathrm{L}$ \\
Cadmium & ICP-AES & $2 \mu \mathrm{g} / \mathrm{L}$ \\
Chromium & ICP-AES & $15 \mu \mathrm{g} / \mathrm{L}$ \\
Copper & ICP-AES & $4 \mu \mathrm{g} / \mathrm{L}$ \\
Iron, total & ICP-AES & $30 \mu \mathrm{g} / \mathrm{L}$ \\
Ferrous iron & Colorimetric method & $30 \mu \mathrm{g} / \mathrm{L}$ \\
Lead & ICP-AES & $6 \mu \mathrm{g} / \mathrm{L}$ \\
Lithium & ICP-AES & $3 \mu \mathrm{g} / \mathrm{L}$ \\
Manganese & ICP-AES & $10 \mu \mathrm{g} / \mathrm{L}$ \\
Molybdenum & ICP-AES & $20 \mu \mathrm{g} / \mathrm{L}$ \\
Nickel & ICP-AES & $30 \mu \mathrm{g} / \mathrm{L}$ \\
Strontium & ICP-AES & $3 \mu \mathrm{g} / \mathrm{L}$ \\
Vanadium & ICP-AES & $4 \mu \mathrm{g} / \mathrm{L}$ \\
Zinc & ICP-AES & $20 \mu \mathrm{g} / \mathrm{L}$ \\
\hline & &
\end{tabular}

18 replicate samples collected during the study period are presented in tables 5-7 and are discussed below.

\section{Field Equipment Blanks}

A field equipment blank is a sample prepared using blank (deionized) water that is passed through all the sampling and processing equipment (Spahr and
Boulger, 1997). This type of sample is used to check for potential contamination of the environmental samples during sampling collection, processing, handling, and analysis. Analytical results for the 15 field equipment blanks collected during the study period are listed in table 5. The measured concentrations for most constituents were at or below the detection limits of the analytical methods. Several samples did have detectable concentrations of molybdenum, although most concentrations were less than twice the analytical detection limit.

\section{Concurrent and Sequential Replicates}

Concurrent and sequential replicate samples are collected to estimate variability in the environmental data. In the concurrent replicate, one sample is collected concurrently with the environmental sample by a second collection team or by using a second set of collection equipment. In the sequential replicate, a replicate is collected as close in time as possible to the environmental sample. Each sample is processed through all the normal steps of a regular water-quality sample (Spahr and Boulger, 1997). For each step of sample processing, the environmental sample is processed before the replicate sample. The replicate samples were processed using a clean filter and equipment.

Ten sequential and eight concurrent replicates were collected during the study period. Comparison of analytical results between environmental samples and sequential replicates and between environmental samples and concurrent replicates for major inorganic and trace-element constituents are listed in tables 6 and 7. The sequential replicates were collected using the same sampling team and the same collection procedures and analytical methods discussed previously in this report. The concurrent replicates were collected as part of another USGS data-collection program, and samples were analyzed at the USGS National Water Quality Laboratory in Lakewood, Colorado (Crowfoot and others, 1998; Crowfoot and others, 1999; Crowfoot and others, written commun., 2000). The majority of the differences between the environmental and the sequential replicates agree 

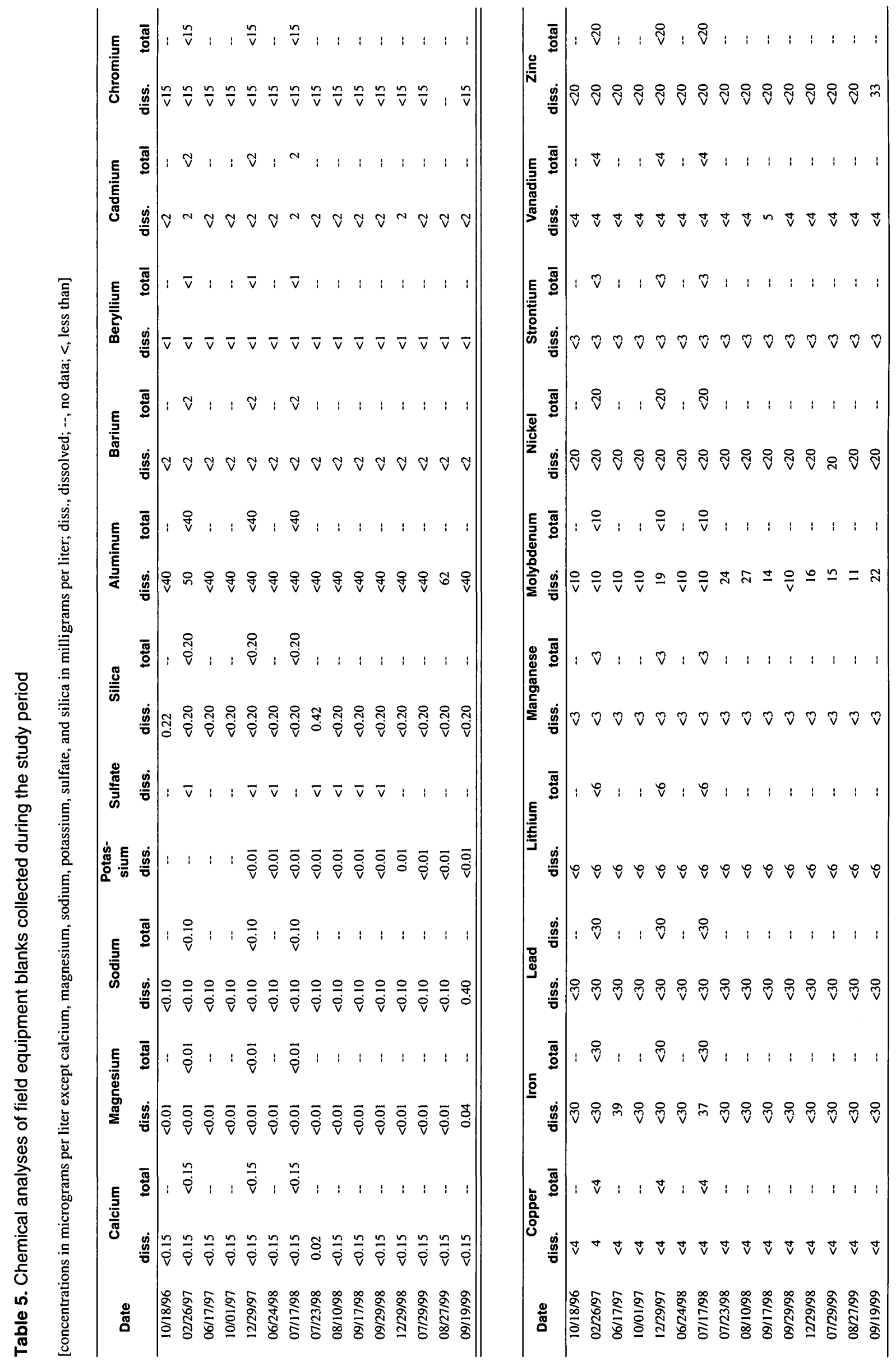

QUALITY ASSURANCE 


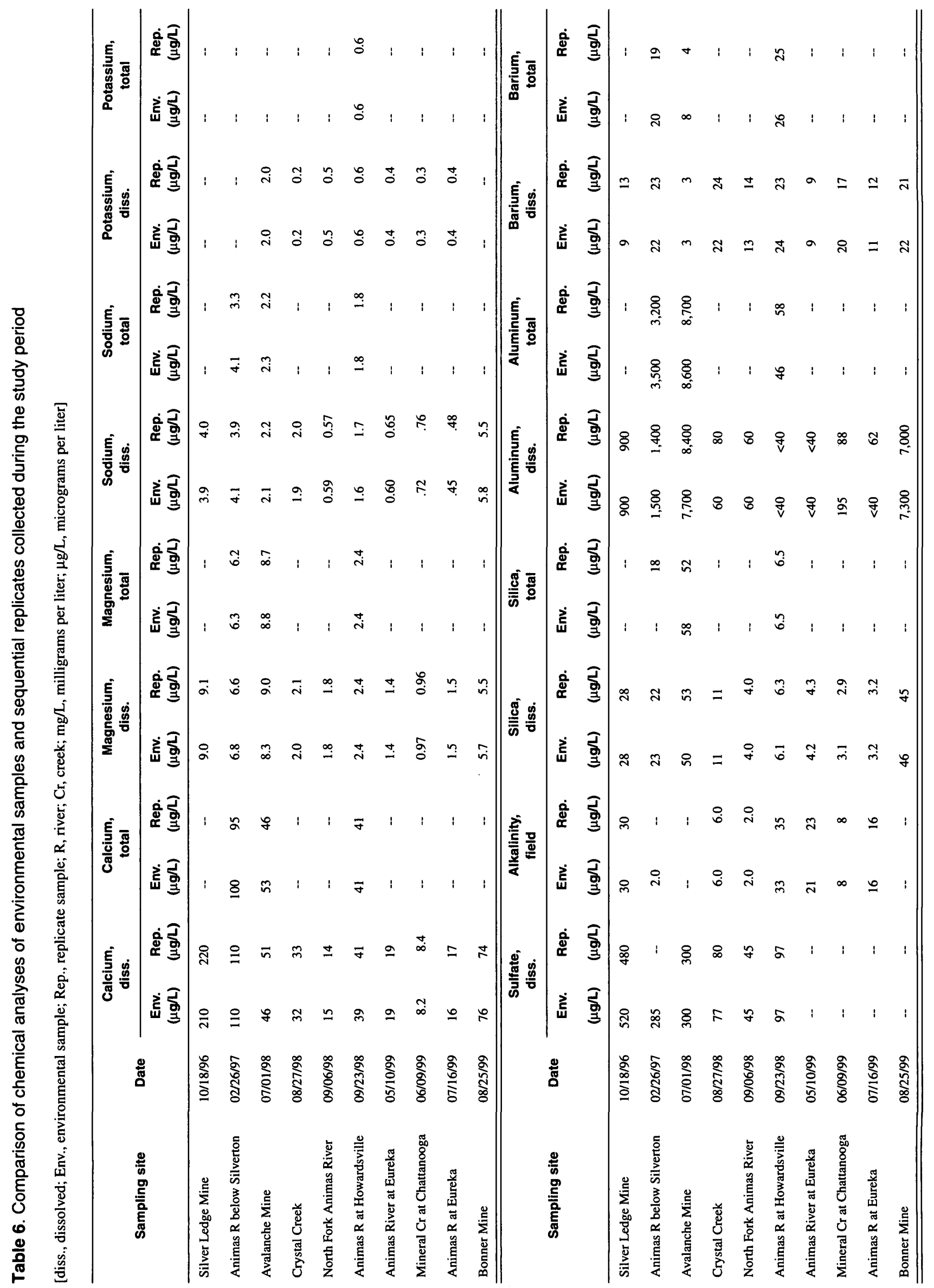




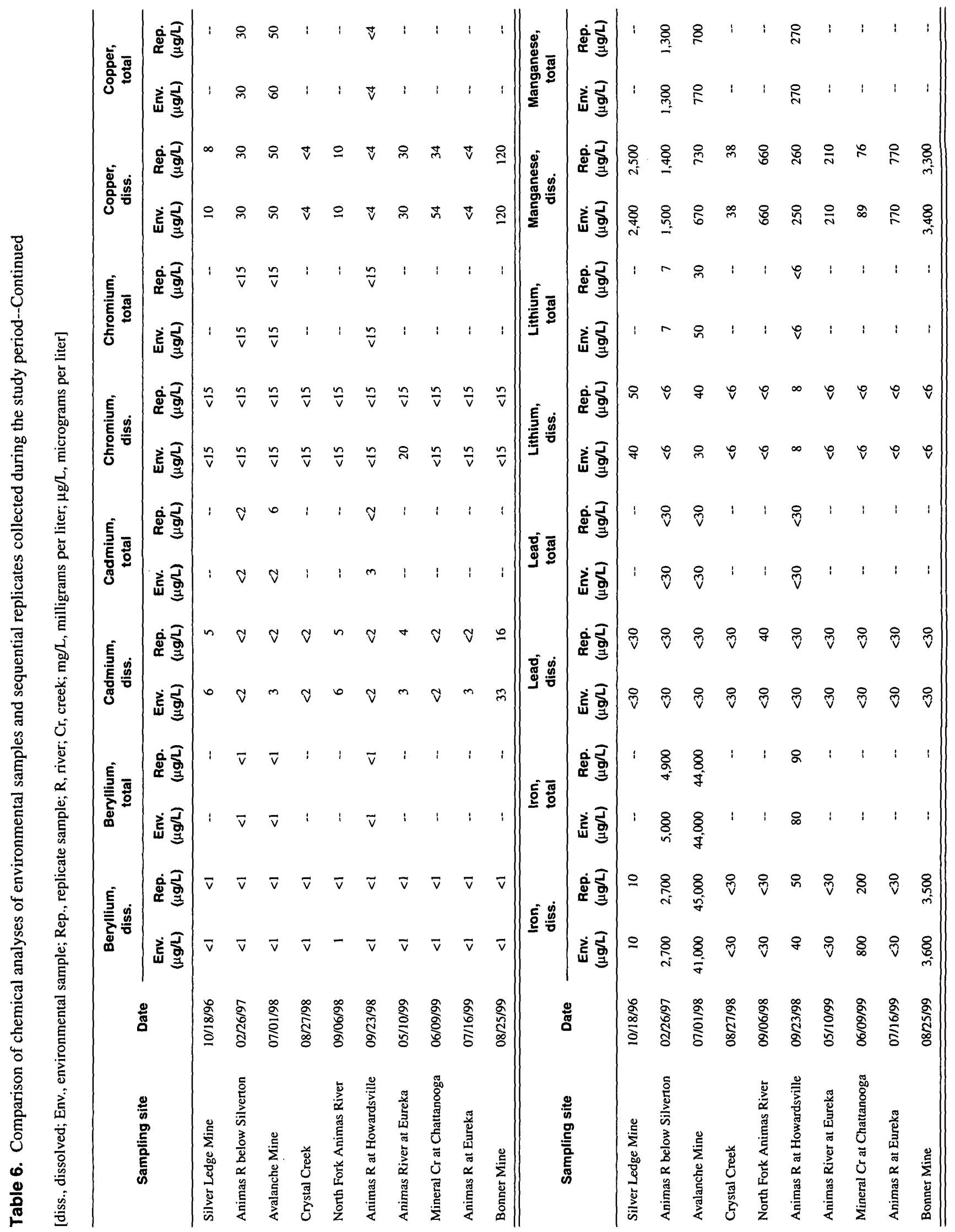




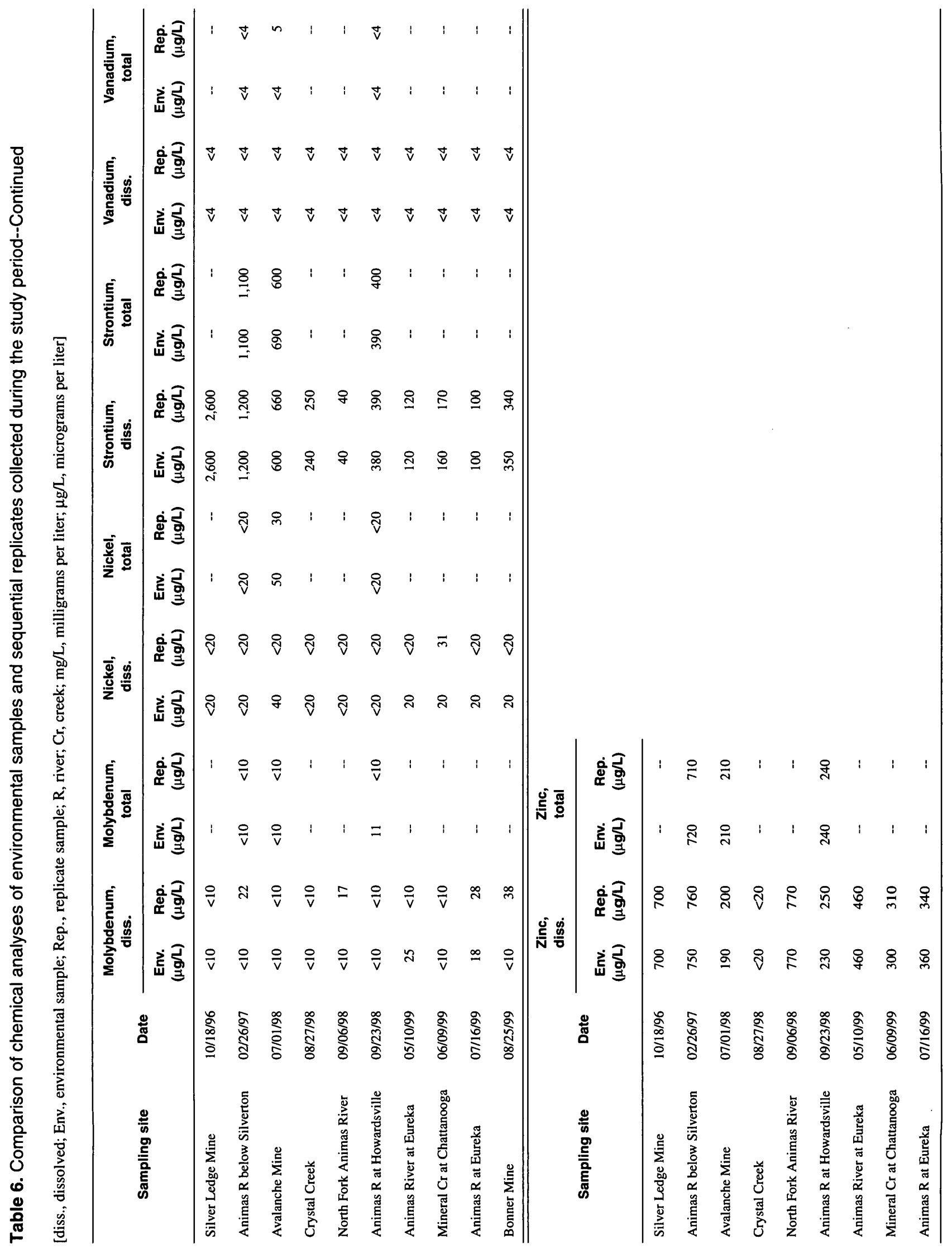




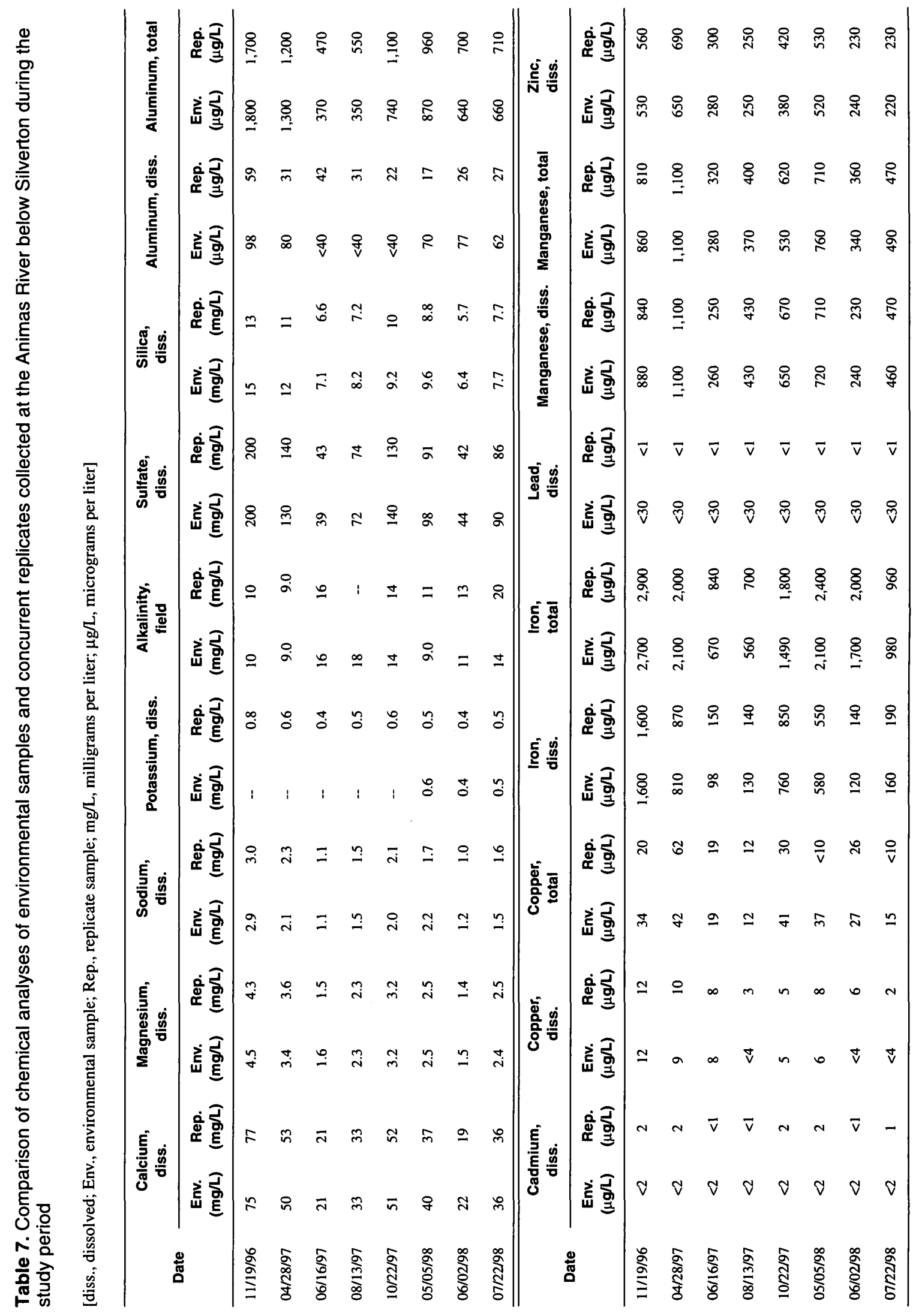


within \pm 10 percent. Many of the trace-element concentrations were less than the analytical detection limit.

\section{REFERENCES}

Bailey, R.G., Avers, P.E., King, T., and McNab, W.H., 1994, Ecoregions and subregions of the United States with supplementary table of map unit descriptions: Washington, D.C., U.S. Department of Agriculture Forest Service, scale 1:7,500,000 (also at URL http://www.fs.fed.us/land/ecosysmgmt/ecoreg1_home. html, accessed March 2000).

Bove, D.J., Kon, K., Slack, Budding, K.E., Slack, J.F., and Yeoman, R.A., 1999, Geochronology and geology of late Oligocene through Miocene volcanism and mineralization in the western San Juan Mountains, Colorado: U.S. Geological Survey Open-File Report 99-347, 35 p.

Burbank, W.S., and Luedke, R.G., 1969, Geology and ore deposits of the Eureka and adjoining districts, San Juan Mountains, Colorado: U.S. Geological Survey Professional Paper 535, 73 p.

Casadevall, T.C., and Ohmoto, H., 1977, Sunnyside mine, Eureka mining district, San Juan County, ColoradoGeochemistry of gold and base metal ore deposition in a volcanic environment: Economic Geology, v. 72, p. 1285-1320.

Crowfoot, R.M., Paillet, A.V., Ritz, G.F., Smith, M.E., Jenkins, R.A., and O'Neill, G.B., 1998, Water resources data, Colorado, water year 1997: U.S. Geological Survey Water-Data Report CO-97-2, $565 \mathrm{p}$.

Crowfoot, R.M., Bruce, N.L., Unruh, J.W., Steinheimer, J.T., Ritz, G.F., Smith, M.E., Jenkins, R.A., and O'Neill, G.B., 1999, Water resources data, Colorado, water year 1998: U.S. Geological Survey Water-Data Report CO-98-2, 569 p.

Edwards, T.K., and Glysson, D.G., 1988, Field methods for measurement of fluvial sediment: U.S. Geological Survey Open-File Report 86-531, 118 p.

Hoffman, G.L., Foilsman, M.J., and Garbarino, J.R., 1996, Methods of analysis by the U.S. Geological Survey National Water Quality Laboratory-In-bottle acid digestion of whole-water samples: U.S. Geological Survey Open-File Report 96-225, 28 p.

Horowitz, A.J., Demas, C.R., and Fitzgerald, K.K., 1994, U.S. Geological Survey protocol for the collection and processing of surface-water samples for the subsequent determination of inorganic constituents in filtered water: U.S. Geological Survey Open-File Report 94-539, 57 p.
Lipman, P.W., Steven, T.A., Luedke, R.G., and Burbank, W.S., 1973, Revised volcanic history of the San Juan, Uncompahgre, Silverton and Lake City calderas in the western San Juan Mountains, Colorado: U.S. Geological Survey, Journal of Research, v. 1, no. 6, 627-642.

Lipman, P.W., Fisher, F.S., Mehnert, H.H., Naeser, C.W., Luedke, R.G., and Steven, T.A., 1976, Multiple ages of mid-Tertiary mineralization and alteration in the western San Juan Mountains, Colorado: Economic Geology, v. 71, p. 571-588.

Long, H.K., and Farrar, J.W., 1995, Report on the U.S. Geological Survey's evaluation program for standard reference samples distributed in May 1995-T-135 (trace constituents). M-134 (major constituents), $\mathrm{N}-45$ (nutrient), $\mathrm{N}-46$ (nutrients), $\mathrm{P}-24$ (low ionic strength), Hg-20 (mercury), and SED-5 (bed material): U.S. Geological Survey Open-File Report 95-395, $135 \mathrm{p}$.

Nimick, D.A., and von Guerard, Paul, 1998, Science for watershed decisions on abandoned mine landsReview of preliminary results: U.S. Geological Survey Open-File Report 98-297, 47 p.

Rantz, S.E., and others, 1982, Measurement and computation of streamflow-Volume 1, Measurement of stage and discharge; Volume 2, Computation of discharge: U.S. Geological Survey Water-Supply Paper 2175, $631 \mathrm{p}$.

Ringrose, C.R., 1982, Geology, geochemistry, and stable isotope studies of a porphyry-style hydrothermal system, West Silverton District, San Juan Mountains, Colorado: Ph.D. dissertation, University of Aberdeen, Scotland, $257 \mathrm{p}$.

Shelton, L.R., 1994, Field guide for collection and processing stream-water samples for the National Water-Quality Assessment Program: U.S. Geological Survey Open-File Report 94-455, 42 p.

Spahr, N.E., and Boulger, R.W., 1997, Interim results of quality-control sampling of surface water for the Upper Colorado River National Water-Quality Assessment Study Unit, water years 1995-96: U.S. Geological Survey Water-Resources Investigations Report 97-4227, 34 p.

Ward, J.R., and Hair, C.A., 1990, Methods for collection and processing of surface-water and bed-material samples for physical and chemical analyses: U.S. Geological Survey Open-File Report 90-140, 71 p.

Wilde, F.D., and Radtke, D.B., 1998, National field manual for collection of water-quality data: U.S. Geological Survey Techniques of Water-Resources Investigations, book 9, chap. A6.

Wirt, Laurie, Leib, K.J., Bove, D.J., Mast, M.A., Evans, J.B., and Meeker, G.P., 1999, Loads of chemical constituents during base-flow and storm-runoff conditions near historical abandoned mines in Prospect Gulch, upper Animas watershed, southwestern Colorado: U.S. Geological Survey Open-File Report 99-0159, 35 p. 
